\title{
Device-Centric Distributed Antenna Transmission: Secure Precoding and Antenna Selection with Interference Exploitation
}

\author{
Zhongxiang Wei, Member, IEEE, Christos Masouros, Senior Member, IEEE
}

\begin{abstract}
We address physical layer security in distributed antenna (DA) systems, where eavesdroppers (Eves) can intercept the information transmitted for the intended receiver (IR). To realize a device-centric, power-efficient and physical layer security-aware system, we aim at minimizing power consumption by jointly designing DA selection and secure precoding. Different from the conventional artificial noise (AN)-aided secure transmission, where $\mathrm{AN}$ is treated as an undesired element for the IR, we design AN such that it is constructive to the IR while keeping destructive to the Eves. Importantly, we investigate two practical scenarios, where the IR and Eves' channel state information (CSI) is imperfectly obtained or the Eves' CSI is completely unknown. To handle the CSI uncertainties, we solve the problems in probabilistic and deterministic robust optimizations respectively, both satisfying the IR' signal-to-interference-and-ratio (SINR) requirement by use of constructive $\mathrm{AN}$ and addressing security against the Eves. Simulation results demonstrate our algorithms consume much less power compared to the centralized antenna (CA) systems with/without antenna selection, as well as the DA systems with conventional AN processing. Last but not least, by the proposed algorithms, the activation of DAs closely relates to devices' locations and quality-of-service $(\mathrm{QoS})$ requirements, featuring a device-centric and on-demand structure.
\end{abstract}

Index Terms-Distributed antenna, Antenna selection, Secure precoding, Robust optimization, Constructive artificial noise

\section{INTRODUCTION}

Wireless Communications for the future Internet of Things (IoT) and Industry 4.0 are required to provide power-efficient transmission together with high security level. In the last decade, centralized multiple-input multiple-output (MIMO) has been considered as a potential technique due to its high throughput and additional spatial diversity for enhancing physical layer security [1] [2] [3]. However, it requires extremely high power consumption caused by the fully activated antennas, and centralized MIMO often suffers from an equal level of path loss (PL) from the antenna array to one device (or user) caused by the co-located antenna (CA) deployment. Besides, edge devices in CA deployment may not be well served due to the severe propagation attenuation, otherwise significant transmission power is required for compensating the propagation loss. To create a device-centric and power

Zhongxiang Wei and Christos Masouros are with the department of Electronic and Electrical Engineering at the University College London, London, UK. Email: \{zhongxiang.wei, c.masouros\}@ucl.ac.uk

This work was supported by the Engineering and Physical Sciences Research Council, UK, under project EP/R007934/1.

This work has been presented in part in the 2019 IEEE International Conference on Acoustics, Speech and Signal Processing (ICASSP'19) and IEEE International Conference on Communications (ICC'19) [1] [2]. efficient structure, distributed antenna (DA) systems have attracted much attention [4].

By geographically distributing the antennas and hence placing them closer to devices, DA systems can reduce the PL impact and obtain blockage-free effect, helping extend coverage and maintain connectivity of networks. Since the contributions of each DA may vary practically due to the location of the devices [5], system power consumption can be significantly reduced by only activating those DAs contributing the most, which also consequently facilitates an on-demand and flexible network structure. The concepts of device-centric DA are particularly suited for communications in industrial environments, where antennas can be carefully planned and distributed in the ceilings of large factories to effectively extend network coverage without crucial increment of power consumption, and are therefore a key contender for industry IoT and industry 4.0 deployments [6]. Besides, DA systems have been extensively deployed as an important part of the landline infrastructure in the USA (e.g., Michigan and Ohio) promulgated by the FCC Pole Attachment Order 11-50 [7]. DA systems have also been considered in $5 \mathrm{G}$ systems to form user-centric manner virtual cells [8], where users can find DAs in its vicinity to communicate with. In indoor environment, DA systems can be applied to provide seamless coverage [9], such as commercial use of WiFi. It was pointed out in the FP7 EARTH project [10] that for a small-scale node (such as DA, femto or pico node), the power consumption is dominated by the power amplifier (PA) and circuit power. Generally, PA power consumption is closely related to the transmission power and drain efficiency at transmitters. On the other hand, circuit power consumption contains multiple power consuming components. The power consumption of an active DA mainly comes from digital/analog converter, ana$\log$ /digital converter, optical/electrical converter, up-converter, filter, synthesizer, etc., while the power consumption of one DA can be significantly reduced by switching it off [11].

Nevertheless, it should be noticed that due to the proximity to transmitting antennas, it is also easier for potential eavesdroppers (Eves) to obtain the signal transmitted to the intended device' receiver (IR), and physical layer security issue in DA systems becomes more challenging. In the past decade, physical layer security has been extensively investigated as a complement to secure wireless communications. For the nonartificial noise (AN) aided scheme, physical layer security is addressed by solely designing beamforming. One can provide a maximized gain towards the IR's channel with no regard 
for leakage into the Eve's channel, or transmit signal towards null space of the Eves with reduced gain into the IR's channel [12]. Besides, CVX or singular value decomposition based precoding can be adopted to make a trade-off between maximizing gain to the IR and steering a null at the Eves [13], which is the secrecy capacity achieving scheme at the cost of high computationally expensive. To further address secrecy outage performance with low power consumption, the authors in [14] [15] investigated the secrecy outage performance with antenna selection while preserving the transmission diversity, benefiting from multiple antennas configuration. Nevertheless, the above approach do not address AN-aided secure transmission, which is the focus of our work. Differently, for the $\mathrm{AN}$-aided secure transmission, $\mathrm{AN}$ is generated at transmitter together with confidential message to jam potential Eves. When the Eves channel state information (CSI) is unknown at transmitter, the $\mathrm{AN}$ is produced such that it lies in the null space of the IR channel [16] [17]. Since the potential Eves channel is different from that of IRs, the isotropic AN could degrade the potential Eves receiving performance while imposing minimal effect on the IRs. Based on the principle, optimal power allocation [18], delay minimization [19], and PHY security with randomly distributed Eves [20] have been investigated. When the Eves' CSI is known at the transmitter, AN could be injected to the direction of the Eves in a spatial manner, which is more efficient than the isotropic manner [21] [22]. Based on the AN-aided secure scheme, secrecy rate [23] [24], outage probability [25] and power consumption [3] [22] have been addressed recently. On the specific topic of secure transmission in DA systems, the authors in [26] investigated SINR maximization problem for DA systems, where AN is generated by DAs to interfere the Eves. The authors in [27] maximized the ergodic secrecy rate in DA systems, where AN and signal are jointly designed and transmitted at each DA. In [3], the power minimization problem was demonstrated for DA systems, where the potential Eves are considered as idle IRs and scavenge energy from AN. It is worth noting that, regardless of isotropic or spatial AN transmission, AN is treated as an undesired element at the IR and its leakage effect needs to be minimized [28].

The aforementioned research, however, treated $\mathrm{AN}$ as a catastrophic element and mitigated the effect of $\mathrm{AN}$ at the IR as much as possible. If AN can be carefully designed at the transmitter, it may be beneficial to the IR in terms of improving signal-to-interference-plus-noise ratio (SINR) based on the concept of constructive interference (CI). The concept of CI was firstly introduced by [29] in code division multiple access. Then a rotated zero-forcing (ZF) precoding scheme was proposed in [30], and [31] further proposed that all the interference can be constructive by designing precoding in symbol level. Recently, the concept of CI was applied into cognitive radio [32], large-scale multi-input multi-output (MIMO) [33], multiuser multi-input single-output (MISO) [34] [35] [36], and wireless power transfer [37]. Based on the aforementioned work in CI, the authors in [38] proposed a scheme to utilize $\mathrm{AN}$ in a CA system. However, the fully activated antennas in [38] lead to enormous power consumption.

Motivated by the aforementioned issues, in this paper, we present joint design of DA selection and secure precoding to minimize total power consumption, subjected to physical layer security constraints. Importantly, we consider two practical scenarios and solve the problems from two different prospectives of robust optimizations, namely probabilistic and deterministic robust optimizations. Our contributions are summarized in the following:

1) We investigate the power minimization problems under the IR's QoS requirement and physical layer security constraints against the Eves. DA selection and precoding are jointly designed to fully utilize the additional degrees of freedom in antennas' activation/deactivation and beneficial effect of AN, which significantly reduce the total power consumption yet maintaining the IR's SINR and security constraints against the Eves.

2) We exploit joint DA selection and robust precoding in two practical scenarios: first, when the IR and Eves' CSI is imperfectly obtained, and second, when the Eves' CSI is completely unknown. Then we investigate the total power minimization problems for the two scenarios in probabilistic and deterministic manners, respectively. In the first scenario, the IR's SINR constraint and security against the Eves are issued by chance constrained formulations from the prospective of probabilistic robust optimization, while the IR's SINR constraint and security against the Eves are guaranteed with all the CSI uncertainties from the prospective of deterministic robust optimization. On the other hand, when the Eves' CSI is completely unknown at the transmitter side in the second scenario, the IR's SINR requirement is addressed by the probabilistic or deterministic robust optimization, while the security towards the Eves is addressed by confining a minimum power level of AN.

3) Four corresponding low-complexity algorithms are proposed to minimize total power consumption for the two scenarios, in terms of probabilistic and deterministic manners. At the same time, AN is kept constructive to the IR whereas destructive to the Eves. Complexities of the algorithms are analytically demonstrated.

4) A device-centric network structure is demonstrated by the proposed schemes, compared to the CA counterpart. Explicitly, the working status of each DA is flexibly determined by the IR and Eves' positions to provide on-demand services: the DAs close to devices have higher probabilities of activation, while the DAs far from devices have higher probabilities of being idle for saving power. Furthermore, the power consumption of the DA deployment remains low regardless of the devices' positions and power-efficient transmission is always featured, while the power consumption of the CA deployment demonstrates a significant increment when the devices move to edge area.

\section{System Model And Constructive Interference}

In this section, system model is introduced in II-A and the concept of CI is briefly discussed in II-B. 
TABLE I. Some notations and symbols used in this paper.

\begin{tabular}{|c|c|}
\hline Symbols & Notations \\
\hline$|\cdot|$ and $\|\cdot\|_{p}$ & $\begin{array}{l}\text { absolute value of a complex scalar and p-norm } \\
\text { of a vector or a matrix }\end{array}$ \\
\hline $\begin{array}{l}\boldsymbol{A}^{H} \quad \boldsymbol{A}^{T}, \quad \operatorname{Tr}(\boldsymbol{A}) \\
\operatorname{Rank}(\boldsymbol{A}) \text { and } \boldsymbol{A} \succeq 0\end{array}$ & $\begin{array}{l}\text { Hermitian transpose, transpose, trace, rank of } \\
\text { matrix } \boldsymbol{A} \text {, and positive semi-definite matrix }\end{array}$ \\
\hline $\operatorname{diag}(\boldsymbol{A})$ and $\operatorname{diag}(\boldsymbol{a})$ & $\begin{array}{l}\text { returns a diagonal matrix with diagonal elements } \\
\text { from matrix } \boldsymbol{A} \text {, and stacks the elements of vector } \\
\boldsymbol{a} \text { into a diagonal matrix }\end{array}$ \\
\hline $\boldsymbol{I}_{n}$ & $n$-by- $n$ identity matrix \\
\hline$\Re$ and $\Im$ & real part and imaginary parts \\
\hline $\mathbb{C}^{N \times M}$ & sets of all $N \times M$ matrices \\
\hline $\mathbb{H}^{N \times M}$ & Hermitian matrices with complex entries \\
\hline$M, N$ and $K$ & constellation size, the number of DAs and Eves \\
\hline$\eta_{d}$ and $\eta_{k}$ & $\begin{array}{l}\text { SINR outage probabilities of the IR and } k \text {-th } \\
\text { Eve for probabilistic robust optimization }\end{array}$ \\
\hline $\begin{array}{l}p_{o f f}, \quad p_{o n}, p_{D A}, \\
p_{A N} \text { and } \alpha\end{array}$ & $\begin{array}{l}\text { power consumption of sleeping DA and active } \\
\text { DA, maximum transmission power of each DA, } \\
\text { minimum power constraint of } \mathrm{AN} \text {, and drain } \\
\text { efficiency at each DA }\end{array}$ \\
\hline$\varphi$ & penalty factor \\
\hline $\boldsymbol{w} \in \mathbb{C}^{N \times 1}$ and $w_{n}$ & $\begin{array}{l}\text { precoding vector and precoding weight of the } \\
n \text {-th DA }\end{array}$ \\
\hline $\boldsymbol{z} \in \mathbb{C}^{N \times 1}$ and $z_{n}$ & AN vector and AN weight of the $n$-th DA \\
\hline $\boldsymbol{u} \in \mathbb{C}^{N \times 1}$ & equivalent transmission vector \\
\hline $\boldsymbol{t} \in \mathbb{C}^{N \times 1}$ and $t_{n}$ & antenna selection vector and the $n$-th DA's status \\
\hline 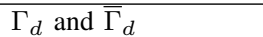 & Receive SINR and SINR requirement of the IR \\
\hline $\bar{\Gamma}_{k}$ and $\bar{\Gamma}_{k}$ & $\begin{array}{l}\text { Receive SINR and SINR requirement of the } k \text {-th } \\
\text { Eve for addressing PHY security }\end{array}$ \\
\hline $\begin{array}{l}n_{d}, \quad \text { and } n_{k} \sim \\
\mathcal{C N}\left(0, \sigma_{n}^{2}\right)\end{array}$ & $\begin{array}{l}\text { Additive white Gaussian noises (AWGN) at the } \\
\text { IR and the } k \text {-th Eve with variance } \sigma_{n}^{2}\end{array}$ \\
\hline $\begin{array}{l}\boldsymbol{h}_{d} \in \mathbb{C}^{N \times 1}, \hat{\boldsymbol{h}}_{d} \in \\
\mathbb{C}^{N \times 1}\end{array}$ & $\begin{array}{l}\text { true channel, estimated channel between the } \\
\text { DAs and IR }\end{array}$ \\
\hline $\begin{array}{l}\boldsymbol{e}_{d} \quad \in \quad \mathbb{C}^{N \times 1} \\
{\left[\boldsymbol{e}_{d}\right]_{n} \sim \mathcal{C N}\left\{0, \sigma_{d}^{2}\right\}}\end{array}$ & $\begin{array}{l}\text { estimation error vectors, and normal distributed } \\
\text { channel estimation error between the } n \text {-th DA } \\
\text { and IR, with variance } \sigma_{d}^{2}\end{array}$ \\
\hline $\begin{array}{l}\boldsymbol{h}_{k} \in \mathbb{C}^{N \times 1}, \hat{\boldsymbol{h}}_{k} \in \\
\mathbb{C}^{N \times 1}\end{array}$ & $\begin{array}{l}\text { true channel and estimated channel between DAs } \\
\text { and the } k \text {-th Eve }\end{array}$ \\
\hline $\begin{array}{l}\boldsymbol{e}_{k} \in \underset{\mathbb{C}^{N \times 1}}{ } \quad \in \quad \\
{\left[\boldsymbol{e}_{k}\right]_{n} \sim \mathcal{C N}\left\{0, \sigma_{k}^{2}\right\}}\end{array}$ & $\begin{array}{l}\text { estimation error vectors between DAs and the } \\
k \text {-th Eve, and normal distributed channel esti- } \\
\text { mation error between the } n \text {-th DA and the } k \text {-th } \\
\text { DA, with variance } \sigma_{k}^{2}\end{array}$ \\
\hline
\end{tabular}

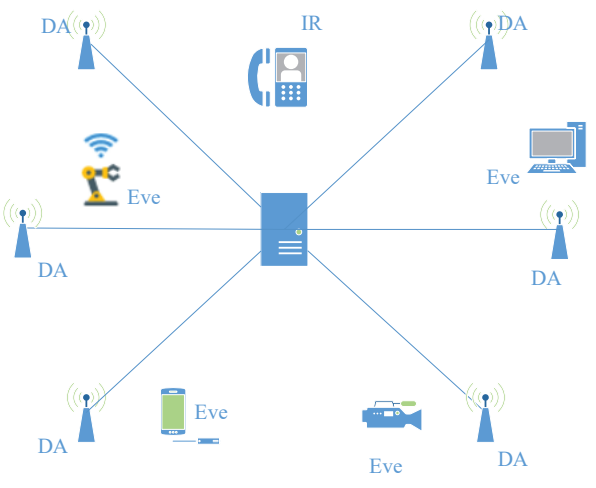

Fig. 1. Illustration of system model, where DAs are geographically positioned.

\section{A. System Model}

We consider a DA system at downlink, which is depicted in Fig. 1. All DA ports are connected to the central unit through a noise-free wired front-haul for cooperative communications. Central unit is equipped with $N$ DAs transmitting confidential message to the IR in the presence of $K$ possible Eves. We focus on secure precoding design with passive Eves, where the Eves only intercept confidential message but do not actively launch attack [14]-[28] ${ }^{1}$. The IR and Eves are all equipped with single antenna for simplicity. CSI is obtained by channel estimation in the training phase, based on channel reciprocity as in [4] [39]. Without loss of generality, we assume that all the DAs share the same drain efficiency and their PAs work in the linear region. Per-DA power constraint is applied, which is essentially different from joint power constraint in CA systems.

\section{B. Constructive Interference}

By the concept of $\mathrm{CI}$, interference pushes the received signals away from the detection threshold [31]. The increased distance to the detection threshold can effectively improve the receiving performance. Denote $\boldsymbol{w} \in \mathbb{C}^{N \times 1}$ and $\boldsymbol{z} \in \mathbb{C}^{N \times 1}$ as precoding vector and $\mathrm{AN}$ at the transmitter side. Without loss of generality, QPSK is employed as the constellation scheme and the secure precoding design with generic constellation schemes is discussed in section V. Denote $x_{d}=d e^{j \phi_{d}}$ as the information-bearing symbol transmitted for the IR. The received signal at the IR and the $k$-th Eve can be calculated

${ }^{1}$ With active Eves, there has been extensive research focusing on device authentication [40] [41], detecting active attacks [42] and offering countermeasures to guarantee secret communications [43] [44]. Higher layer authentication works as a digital signature to verify the validity of a legitimate nodes identity from unauthorized nodes before establishing legitimate communications links for data transmission [43] [45] [46]. In this paper we focus on secure precoding design for already authenticated links to enhance security against potential eavesdroppers [14]-[28]. In addition to MAC authentication, there are other authentication schemes, including network-layer authentication [47], transport-layer authentication [48] and application layer authentication [46]. In particular, the key point of PHY-authentication is to recognize the PHY identities of wireless devices for authentication purpose [49] [50], such as CSI-based authentication (the propagation characteristics of wireless channels) [51] [52] [53], RF recognition approaches (also known as device fingerprints) [54], and wiretap code-based authentication [55]. Another line of research is to model the transmitter and malicious jammer as players in a game-theoretic formulation with the mutual information as the payoff function, and to identify the optimal transmit strategies for both parties. Taking ergodic MIMO secrecy rate as payoff function, [57] employed Nash equilibria to optimize the payoff function. Based on the stochastic game theory, the transmitter in [56] chooses among transmitting, remaining silent or acting as a jammer for secure transmission. Based on mixed-strategy Nash equilibria, the authors of [58] conducted relay selection at uplink transmission to improve transmission rate. Besides, the security design with compromised base station involves intrusion detection at higher layer [59], or distributed storage coding (also known as regenerating codes) design [60]. The former aims to detect the compromised nodes while the latter is normally employed for distributed storage systems (DDS). That is, it is desired that legitimate users be able to reconstruct the original files by retrieving data from a subset of storage nodes, while protecting it from being reconstructed by Eves, even with compromised storage nodes [60]. Please note that the essence of DA systems is completely different from that of DDS. For DA systems, each DA only serves as geographically positioned remote radio head without ability of data storage, while the essence of distributed data systems is that chunks of data files are stored across different storage sites based on cloud technology. Hence, the motivation of DA is to extend network coverage and enable the user-centric network structure, while distributed data systems are employed for distributing data into different storage sites for load balancing. 


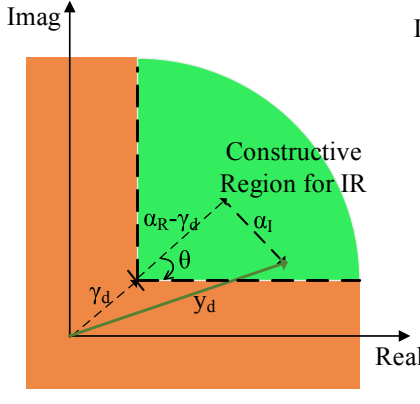

(a)

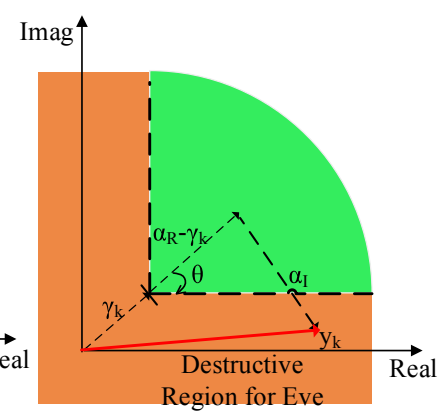

(b)
Fig. 2. Constructive and destructive AN for the IR and Eves with QPSK. (a) Constructive AN pushes the IR's received symbols towards the constructive region, where $\alpha_{R}=\Re\left(\boldsymbol{h}_{d}\left(\boldsymbol{w}+\boldsymbol{z} e^{-j \phi_{d}}\right)\right), \alpha_{I}=\Im\left(\boldsymbol{h}_{d}\left(\boldsymbol{w}+\boldsymbol{z} e^{-j \phi_{d}}\right)\right)$, and $\gamma_{d}=\sigma_{n} \sqrt{\Gamma_{d}}$. (b) Destructive AN pushes the Eves' received symbols towards to destructive region, where $\alpha_{R}=\Re\left(\boldsymbol{h}_{k}\left(\boldsymbol{w}+\boldsymbol{z} e^{-j \phi_{d}}\right)\right), \alpha_{I}=$ $\Im\left(\boldsymbol{h}_{k}\left(\boldsymbol{w}+\boldsymbol{z} e^{-j \phi_{d}}\right)\right)$, and $\gamma_{k}=\sigma_{n} \sqrt{\Gamma_{k}}$.

as $y_{d}=\boldsymbol{h}_{d}^{T}\left(\boldsymbol{w} x_{d}+\boldsymbol{z}\right)+n_{d}$, and $y_{k}=\boldsymbol{h}_{k}^{T}\left(\boldsymbol{w} x_{d}+\boldsymbol{z}\right)+n_{k}$, where $\boldsymbol{h}_{d} \in \mathbb{C}^{N \times 1}$ denotes the channel conditions between the DAs and the IR. $\boldsymbol{h}_{k} \in \mathbb{C}^{N \times 1}$ denotes the channel conditions between the DAs and the $k$-th Eve. $n_{d} \sim \mathcal{C N}\left(0, \sigma_{n}^{2}\right)$ and $n_{k} \sim \mathcal{C N}\left(0, \sigma_{n}^{2}\right)$ denote the Additive white Gaussian noises (AWGN) at the IR and the $k$-th Eve, respectively. Conventionally, the received SINR at the IR and $k$-th Eve are denoted as

$$
\Gamma_{d}=\frac{\left|\boldsymbol{h}_{d}^{T} \boldsymbol{w}\right|^{2}}{\sigma_{n}^{2}+\left|\boldsymbol{h}_{d}^{T} \boldsymbol{z}\right|^{2}}, \Gamma_{k}=\frac{\left|\boldsymbol{h}_{k}^{T} \boldsymbol{w}\right|^{2}}{\sigma_{n}^{2}+\left|\boldsymbol{h}_{k}^{T} \boldsymbol{z}\right|^{2}},
$$

where $\Gamma_{d}$ and $\Gamma_{k}$ denote the IR and the $k$-th Eve's SINR, respectively. It can be seen from (1) that AN is treated as an undesired element at the IR. By contrast, the principle of constructive AN is to rotate the phase of the AN at transmitter and to align it with the desired signal at the IR. Since the transmitted signal can be also written as $\left(\boldsymbol{w}+\boldsymbol{z} e^{-j \phi_{d}}\right) x_{d}$, by exploiting the geometrical interpretation in Fig. 2, generating constructive $\mathrm{AN}$ at the IR is equivalent to satisfying the following equation

$$
\begin{aligned}
& \left|\Im\left\{\boldsymbol{h}_{d}^{T} \boldsymbol{w}+\boldsymbol{h}_{d}^{T} \boldsymbol{z} e^{-j \phi_{d}}\right\}\right| \leq \\
& \quad\left(\Re\left\{\boldsymbol{h}_{d}^{T} \boldsymbol{w}+\boldsymbol{h}_{d}^{T} \boldsymbol{z} e^{-j \phi_{d}}\right\}-\gamma_{d}\right) \cdot \tan \theta,
\end{aligned}
$$

where $\theta=\pi / M, M$ is constellation size [38] and $\gamma_{d}=$ $\sigma_{n} \sqrt{\Gamma_{d}}$, as shown in Fig. 2. It can be seen from (2) that the AN becomes a beneficial element to the IR and the IR' SINR $\Gamma_{d}$ is also embedded, indicating that generating constructive AN and satisfying the IR's SINR requirement are both guaranteed. Since the AN contributes to the useful signal power, the received SINR of the IR becomes

$$
\Gamma_{d}=\frac{\left|\boldsymbol{h}_{d}^{T}\left(\boldsymbol{w}+\boldsymbol{z} e^{-j \phi_{d}}\right)\right|^{2}}{\sigma_{n}^{2}},
$$

where transmission power can be efficiently reduced to achieve a target SINR.

\section{Power Efficient DA Selection and Secure PRECODING WITH IMPERFECT CSI}

In Section III, we investigate power efficient design with imperfect CSI, where the channels are given as $\boldsymbol{h}_{d}=\hat{\boldsymbol{h}}_{d}+\boldsymbol{e}_{d}$ and $\boldsymbol{h}_{k}=\hat{\boldsymbol{h}}_{k}+\boldsymbol{e}_{k}, \forall k \in K . \hat{\boldsymbol{h}}_{d} \in \mathbb{C}^{N \times 1}$ denotes the estimated CSI between the DAs and the IR with estimation error $\boldsymbol{e}_{d} \in \mathbb{C}^{N \times 1}$. $\hat{\boldsymbol{h}}_{k} \in \mathbb{C}^{N \times 1}$ denotes the estimated CSI between the DAs and the $k$-th Eve with estimation error $\boldsymbol{e}_{k} \in \mathbb{C}^{N \times 1}$. By classic minimum mean square error (MMSE) channel estimation, the channel estimation error can be modeled by a standard normal distributed variable or an error-bounded variable. Explicitly, it relates to the channel estimation setup, e.g., power of pilot signal. To fully exploit the power efficient design with the uncertainties (channel estimation error), we handle the optimization problem in probabilistic and deterministic manners, respectively.

\section{A. Probabilistic Robust Optimization}

In subsetion III-A, we present the precoding design with the normal distributed CSI error. Without loss of generality, the channel estimation error of the IR and the $k$-th Eve are written as $\left[\boldsymbol{e}_{d}\right]_{n} \sim \mathcal{C N}\left\{0, \sigma_{d}^{2}\right\}$ and $\left[\boldsymbol{e}_{k}\right]_{n} \sim \mathcal{C N}\left\{0, \sigma_{k}^{2}\right\}, \forall k \in$ $K, n \in N$, respectively. In this subsection, a so-called DAimperfect-prob optimization problem is firstly formulated by P1(a) (Eq. 4), and then is transformed into P1(b) (Eq. 15) by transforming the probabilistic constraints $(\mathrm{C} 3)$ and $(\mathrm{C} 4)$ into equivalent linear matrix inequality (LMI). Finally, the problem is given by $\mathrm{P} 1$ (d) (Eq. 20) after handling the binary variables in antenna selection.

1) Problem Formulation: Define precoding vector $\boldsymbol{w} \in$ $\mathbb{C}^{N \times 1}$, whose $n$-th element $w_{n}$ represents the precoding weight at the $n$-th DA. Define AN vector $\boldsymbol{z} \in \mathbb{C}^{N \times 1}$, whose element $z_{n}$ represents the AN generated at the $n$-th DA. Define DA selection vector $\boldsymbol{t}$, whose element $t_{n}=\{0,1\}$ means the $n$-th DA is deactivated or activated, respectively. Taking advantage of CI, AN is properly rotated such that it contributes to the received signal power at the IR while remaining destructive to the Eves. To minimize the total power consumption, we jointly optimize precoding $\boldsymbol{w}, \mathrm{AN} z$ and DA selection vector $\boldsymbol{t}$. Accordingly, the problem is formulated as

$$
\begin{aligned}
& P 1(a) \text { (DA - imperfect - prob): } \\
& \underset{\boldsymbol{w}, \boldsymbol{z}, t}{\operatorname{argmin}} \frac{\left\|\boldsymbol{w}+\boldsymbol{z} e^{-j \phi_{d}}\right\|^{2}}{\alpha}+\sum_{n=1}^{N}\left(t_{n} p_{o n}+\left(1-t_{n}\right) p_{o f f}\right), \\
& \text { s.t }(C 1): 0 \leq\left|w_{n}+z_{n} e^{-j \phi_{d}}\right|^{2} \leq t_{n} p_{D A}, \forall n \in N, \\
& (C 2): t_{n}=\{0,1\}, \forall n \in N,(C 3): \operatorname{Pr}\left\{\Gamma_{d} \geq \bar{\Gamma}_{d}\right\} \geq \eta_{d}, \\
& (C 4): \operatorname{Pr}\left\{\Gamma_{k} \leq \bar{\Gamma}_{k}\right\} \geq \eta_{k}, \forall k \in K,
\end{aligned}
$$

where $\alpha$ is the drain efficiency of the DAs. $p_{D A}$ denotes the maximum available transmission power at each DA. $p_{o n}$ and $p_{\text {off }}$ represent the power consumption of each activated/deactivated DA, respectively. $\bar{\Gamma}_{d}$ and $\bar{\Gamma}_{k}$ are the SINR requirement for the IR and physical layer security against the $k$-th Eve. $\eta_{d}$ and $\eta_{k}$ denote the probabilistic thresholds for the IR and the $k$-th Eve, respectively. Evidently, $(C 1)$ imposes individual transmission power constraint at each DA, which is different from the joint transmission power constraint in CA systems. $(C 2)$ constrains the selection vector to binary (on/off) elements. $(C 3)$ and $(C 4)$ guarantee the SINR constraint at the IR and address security against the Eves with probabilities $\eta_{d}$ and $\eta_{k}, \forall k \in K$. 
2) Solution to the Problem: We now handle the probabilistic constraints $(C 3)$ and (C4). Define $\boldsymbol{u}=\boldsymbol{w}+\boldsymbol{z} e^{-j \phi_{d}}$. By exploiting trigonometry in Eq. (2), generating constructive AN for the IR and satisfying the IRs SINR requirement are both guaranteed. Under the provision of (2), the probabilistic constraint $(\mathrm{C} 3)$ is equivalent to

$$
(C 3): \operatorname{Pr}\left\{\left|\Im\left\{\boldsymbol{h}_{d}^{T} \boldsymbol{u}\right\}\right| \leq\left(\Re\left\{\left(\boldsymbol{h}_{d}^{T} \boldsymbol{u}\right\}-\sigma_{n} \sqrt{\bar{\Gamma}_{d}}\right) \tan \theta\right\} \geq \eta_{d} .\right.
$$

Considering the effect of imperfect channel estimation, we have

$$
\begin{aligned}
(C 3): \operatorname{Pr}\left\{\left|\Im\left\{\left(\hat{\boldsymbol{h}}_{d}+\boldsymbol{e}_{d}\right)^{T} \boldsymbol{u}\right\}\right| \leq\right. \\
\left.\quad\left(\Re\left\{\left(\hat{\boldsymbol{h}}_{d}+\boldsymbol{e}_{d}\right)^{T} \boldsymbol{u}\right\}-\sigma_{n} \sqrt{\bar{\Gamma}_{d}}\right) \tan \theta\right\} \geq \eta_{d} .
\end{aligned}
$$

Decomposing the real part and imaginary parts, (6) is equivalent to the two constraints in (7) ${ }^{2}$

$$
\left\{\begin{array}{l}
\operatorname{Pr}\left\{\boldsymbol{a}_{\boldsymbol{d}, \mathbf{1}}^{T}\left[\boldsymbol{u}_{R}^{T}, \boldsymbol{u}_{I}^{T}\right]^{T} \leq-\sigma_{n} \sqrt{\bar{\Gamma}_{d}} \tan \theta\right\} \geq \eta_{d}, \\
\operatorname{Pr}\left\{\boldsymbol{a}_{\boldsymbol{d}, \mathbf{2}}^{T}\left[\boldsymbol{u}_{R}^{T}, \boldsymbol{u}_{I}^{T}\right]^{T} \leq-\sigma_{n} \sqrt{\overline{\bar{\Gamma}_{d}}} \tan \theta\right\} \geq \eta_{d},
\end{array}\right.
$$

where $\boldsymbol{a}_{d, 1}=\left[\left(\hat{\boldsymbol{h}}_{I, d}-\hat{\boldsymbol{h}}_{R, d} \tan \theta+\boldsymbol{e}_{I, d}-\boldsymbol{e}_{R, d} \tan \theta\right)^{T},\left(\hat{\boldsymbol{h}}_{R, d}+\right.\right.$ $\left.\left.\hat{\boldsymbol{h}}_{I, d} \tan \theta+\boldsymbol{e}_{R, d}+\boldsymbol{e}_{I, d} \tan \theta\right)^{T}\right]^{T}$, and $\boldsymbol{a}_{d, 2}=\left[\left(-\hat{\boldsymbol{h}}_{I, d}-\right.\right.$ $\left.\hat{\boldsymbol{h}}_{R, d} \tan \theta-\boldsymbol{e}_{I, d}-\boldsymbol{e}_{R, d} \tan \theta\right)^{T},\left(-\hat{\boldsymbol{h}}_{R, d}+\hat{\boldsymbol{h}}_{I, d} \tan \theta-\boldsymbol{e}_{R, d}+\right.$ $\left.\left.e_{I, d} \tan \theta\right)^{T}\right]^{T}$. We now handle the first inequality in (7). It is easy to obtain that the $N$-dimensional normal distributed vector $\boldsymbol{a}_{d, 1}$ 's expectation is $\overline{\boldsymbol{a}}_{d, 1}=\left[\left(\hat{\boldsymbol{h}}_{I, d}-\right.\right.$ $\left.\left.\hat{\boldsymbol{h}}_{R, d} \tan \theta\right)^{T},\left(\hat{\boldsymbol{h}}_{R, d}+\hat{\boldsymbol{h}}_{I, d} \tan \theta\right)^{T}\right]^{T}$ with covariance matrix $\boldsymbol{\Theta}_{d, 1}=\operatorname{diag}(\underbrace{(1+\tan \theta)^{2} \sigma_{d}^{2}, \ldots,(1+\tan \theta)^{2} \sigma_{d}^{2}}_{2 N})$. Evidently, the inequality can be interpreted as a cumulative probability function (cdf) of a normal distributed variable. By normalizing it into a standard normal distributed variable, we have

$$
\Phi\left(\frac{-\sigma_{n} \sqrt{\bar{\Gamma}_{d}} \tan \theta-\overline{\boldsymbol{a}}_{d, 1}^{T}\left[\boldsymbol{u}_{R}^{T}, \boldsymbol{u}_{I}^{T}\right]^{T}}{\left\|\Theta_{d, 1}^{\frac{1}{2}}\left[\boldsymbol{u}_{R}^{T}, \boldsymbol{u}_{I}^{T}\right]^{T}\right\|_{2}}\right) \geq \eta_{d},
$$

where $\Phi(x)=\frac{1}{\sqrt{2 \pi}} \int_{-\infty}^{x} e^{\frac{-t^{2}}{2}} \mathrm{~d} t$. Defining $\Phi^{-1}(\cdot)$ as the inverse function, (8) is derived into

$$
\overline{\boldsymbol{a}}_{d, 1}^{T}\left[\boldsymbol{u}_{R}^{T}, \boldsymbol{u}_{I}^{T}\right]^{T}+\Phi^{-1}\left(\eta_{d}\right)\left\|\Theta_{d, 1}^{\frac{1}{2}}\left[\boldsymbol{u}_{R}^{T}, \boldsymbol{u}_{I}^{T}\right]^{T}\right\|_{2} \leq-\sigma_{n} \sqrt{\bar{\Gamma}_{d}} \tan \theta
$$

Similarly, the second equation in (7) can be given as

$$
\overline{\boldsymbol{a}}_{d, 2}^{T}\left[\boldsymbol{u}_{R}^{T}, \boldsymbol{u}_{I}^{T}\right]^{T}+\Phi^{-1}\left(\eta_{d}\right)\left\|\Theta_{2}^{\frac{1}{2}}\left[\boldsymbol{u}_{R}^{T}, \boldsymbol{u}_{I}^{T}\right]^{T}\right\|_{2} \leq-\sigma_{n} \sqrt{\bar{\Gamma}_{d}} \tan \theta
$$

where $\overline{\boldsymbol{a}}_{d, 2}=\left[\left(-\hat{\boldsymbol{h}}_{I, d}-\hat{\boldsymbol{h}}_{R, d} \tan \theta\right)^{T},\left(-\hat{\boldsymbol{h}}_{R, d}+\hat{\boldsymbol{h}}_{I, d} \tan \theta\right)^{T}\right]^{T}$ and covariance matrix is calculated as $\boldsymbol{\Theta}_{d, 2}=$

${ }^{2} \operatorname{Pr}\{|X| \leq c\} \geq \eta$ is equivalent to $\operatorname{Pr}\{-X \leq c, X \leq c\} \geq \eta$. However, considering the complicated optimization problems, presenting the optimal precoding design with the joint probability may be infeasible. Moreover, the optimal precoding may not exist unless the joint probability satisfying some strict conditions [32]. Hence, to strike a good trade-off between system performance and complexity, we approximate $\operatorname{Pr}\{|X| \leq c\} \geq \eta$ to $\operatorname{Pr}\{X \leq c\} \geq \eta$ and $\operatorname{Pr}\{-X \leq c\} \geq \eta$. More important, given a high value of $\eta$, i.e., $\eta=95 \%$, the approximation is tight and has been widely utilized in robust optimization design [32]. $\operatorname{diag}(\underbrace{(1+\tan \theta)^{2} \sigma_{d}^{2}, \ldots,(1+\tan \theta)^{2} \sigma_{d}^{2}})$. Hence, $\quad(C 3)$ is is

equivalent to the two inequalities

$\left\{\begin{array}{c}\overline{\boldsymbol{a}}_{d, 1}^{T}\left[\boldsymbol{u}_{R}^{T}, \boldsymbol{u}_{I}^{T}\right]^{T}+\Phi^{-1}\left(\eta_{d}\right)\left\|\Theta_{d, 1}^{\frac{1}{2}}\left[\boldsymbol{u}_{R}^{T}, \boldsymbol{u}_{I}^{T}\right]^{T}\right\|_{2} \leq-\sigma_{n} \sqrt{\bar{\Gamma}_{d}} \tan \theta \\ \overline{\boldsymbol{a}}_{d, 2}^{T}\left[\boldsymbol{u}_{R}^{T}, \boldsymbol{u}_{I}^{T}\right]^{T}+\Phi^{-1}\left(\eta_{d}\right)\left\|\Theta_{d, 2}^{\frac{1}{2}}\left[\boldsymbol{u}_{R}^{T}, \boldsymbol{u}_{I}^{T}\right]^{T}\right\|_{2} \leq-\sigma_{n} \sqrt{\bar{\Gamma}_{d}} \tan \theta\end{array}\right.$

We now handle the security constraint in $(C 4)$ under the provision of destructive interference. According to geometrical interpretation, confining the $k$-th Eve in the destructive region equals to satisfying $\left|\Im\left\{\boldsymbol{h}_{k}^{T} \boldsymbol{u}\right\}\right| \geq\left(\Re\left\{\boldsymbol{h}_{k}^{T} \boldsymbol{u}\right\}-\sigma_{n} \sqrt{\bar{\Gamma}_{k}}\right) \tan \theta$. Taking into account of channel estimation error, (C4) can be expanded as

$$
\begin{aligned}
(C 4): \operatorname{Pr}\left\{\left|\Im\left\{\left(\hat{\boldsymbol{h}}_{k}+\boldsymbol{e}_{k}\right)^{T} \boldsymbol{u}\right\}\right| \geq\right. \\
\left.\quad\left(\Re\left\{\left(\hat{\boldsymbol{h}}_{k}+\boldsymbol{e}_{k}\right)^{T} \boldsymbol{u}\right\}-\sigma_{n} \sqrt{\bar{\Gamma}_{d}}\right) \tan \theta\right\} \geq \eta_{k},
\end{aligned}
$$

which can be transformed into a cdf of a standard normal distributed variable as we did in manipulating (5)-(9). Finally, constraint (C4) holds if the two constraints in (13) are simultaneously satisfied.

$$
\begin{aligned}
& \operatorname{Pr}\left\{\boldsymbol{a}_{k, \mathbf{1}}^{T}\left[\boldsymbol{u}_{R}^{T}, \boldsymbol{u}_{I}^{T}\right]^{T} \leq \sigma_{n} \sqrt{\bar{\Gamma}_{k}} \tan \theta\right\} \geq \eta_{k}, \\
& \operatorname{Pr}\left\{\boldsymbol{a}_{\boldsymbol{k}, \mathbf{2}}^{T}\left[\boldsymbol{u}_{R}^{T}, \boldsymbol{u}_{I}^{T}\right]^{T} \leq \sigma_{n} \sqrt{\bar{\Gamma}_{k}} \tan \theta\right\} \geq \eta_{k},
\end{aligned}
$$

where $\boldsymbol{a}_{k, 1}=\left[\left(\hat{\boldsymbol{h}}_{I, k}+\hat{\boldsymbol{h}}_{R, k} \tan \theta+\boldsymbol{e}_{I, k}+\boldsymbol{e}_{R, k} \tan \theta\right)^{T},\left(\hat{\boldsymbol{h}}_{R, k}-\right.\right.$ $\left.\left.\hat{\boldsymbol{h}}_{I, k} \tan \theta+\boldsymbol{e}_{R, k}-\boldsymbol{e}_{I, k} \tan \theta\right)^{T}\right]^{T}$ and $\boldsymbol{a}_{k, 2}=\left[\left(-\hat{\boldsymbol{h}}_{I, k}+\right.\right.$ $\left.\hat{\boldsymbol{h}}_{R, k} \tan \theta-\boldsymbol{e}_{I, k}+\boldsymbol{e}_{R, k} \tan \theta\right)^{T},\left(-\hat{\boldsymbol{h}}_{R, k}-\hat{\boldsymbol{h}}_{I, k} \tan \theta-\boldsymbol{e}_{R, k}-\right.$ $\left.\left.\boldsymbol{e}_{I, k} \tan \theta\right)^{T}\right]^{T}$. Again, by normalizing the two inequalities in (13) into cdf of standard normal distributed variables, we have

$\left\{\begin{array}{l}\overline{\boldsymbol{a}}_{k, 1}^{T}\left[\boldsymbol{u}_{R}^{T}, \boldsymbol{u}_{I}^{T}\right]^{T}+\Phi^{-1}\left(\eta_{k}\right)\left\|\Theta_{k, 1}^{\frac{1}{2}}\left[\boldsymbol{u}_{R}^{T}, \boldsymbol{u}_{I}^{T}\right]^{T}\right\|_{2} \leq \sigma_{n} \sqrt{\bar{\Gamma}_{k}} \tan \theta, \\ \overline{\boldsymbol{a}}_{k, 2}^{T}\left[\boldsymbol{u}_{R}^{T}, \boldsymbol{u}_{I}^{T}\right]^{T}+\Phi^{-1}\left(\eta_{k}\right)\left\|\Theta_{k, 2}^{\frac{1}{2}}\left[\boldsymbol{u}_{R}^{T}, \boldsymbol{u}_{I}^{T}\right]^{T}\right\|_{2} \leq \sigma_{n} \sqrt{\overline{\Gamma_{k}}} \tan \theta,\end{array}\right.$

where $\overline{\boldsymbol{a}}_{k, 1}=\left[\left(\hat{\boldsymbol{h}}_{I, k}+\hat{\boldsymbol{h}}_{R, k} \tan \theta\right)^{T},\left(\hat{\boldsymbol{h}}_{R, k}-\hat{\boldsymbol{h}}_{I, k} \tan \theta\right)^{T}\right]^{T}$ and $\overline{\boldsymbol{a}}_{k, 2}=\left[\left(-\hat{\boldsymbol{h}}_{I, k}+\hat{\boldsymbol{h}}_{R, k} \tan \theta\right)^{T},\left(-\hat{\boldsymbol{h}}_{R, k}-\hat{\boldsymbol{h}}_{I, k} \tan \theta\right)^{T}\right]^{T}$ with covariance matrix $\boldsymbol{\Theta}_{k, 1}=\boldsymbol{\Theta}_{k, 2}=$ $\operatorname{diag}(\underbrace{(1+\tan \theta)^{2} \sigma_{k}^{2}, \ldots,(1+\tan \theta)^{2} \sigma_{k}^{2}}_{2 N})$. After a series

of transformation, the probabilistic constraints $(C 3)$ and $(C 4)$ in $\mathrm{P} 1(\mathrm{a})$ are transformed into the corresponding quadratic constraints in (11) and (14). Hence, P1(a) is re-formulated as

$$
\begin{aligned}
& P 1(b)(\mathrm{DA}-\text { imperfect }- \text { prob) }: \\
& \underset{\boldsymbol{u}, t_{n}, n \in N}{\operatorname{argmin}} \frac{\|\boldsymbol{u}\|^{2}}{\alpha}+\sum_{n=1}^{N}\left(t_{n} p_{o n}+\left(1-t_{n}\right) p_{o f f}\right), \\
& \text { s.t }(C 1): 0 \leq\left|u_{n}\right|^{2} \leq t_{n} p_{D A}, \forall n \in N,(C 3):(11), \\
& \quad(C 2): t_{n}=\{0,1\}, \forall n \in N,(C 4):(14), \forall k \in K .
\end{aligned}
$$

According to Schur Complements, constraints (C3) and (C4) can be further transformed into (16) and (17), respectively, as shown at the top. Defining $\boldsymbol{U}=\boldsymbol{u}^{H} \boldsymbol{u}, \mathrm{P} 1$ (b) (DA-imperfectprob) is further transformed to 


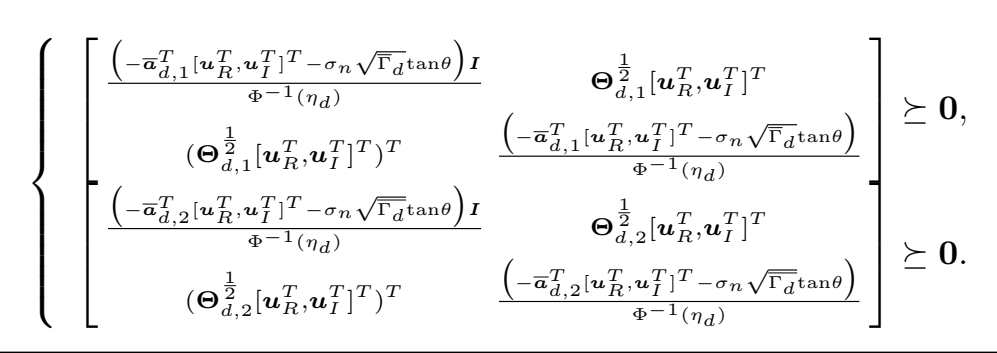

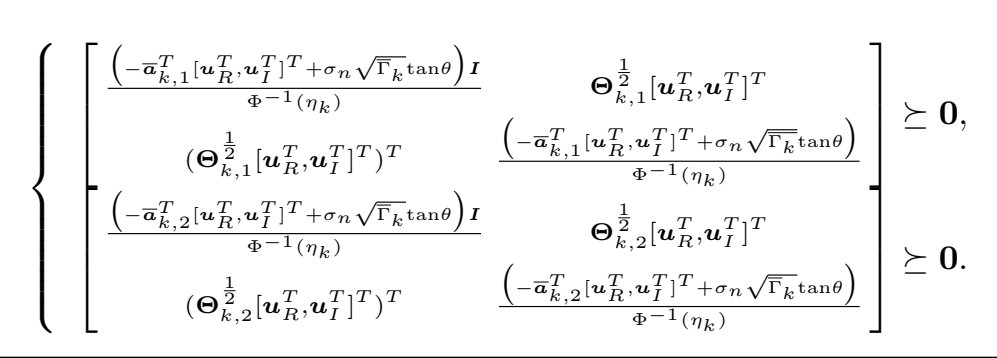

$$
\begin{aligned}
& P 1(c)(\mathrm{DA}-\text { imperfect - prob) : } \\
& \underset{\boldsymbol{u}, \boldsymbol{t}}{\operatorname{argmin}} \frac{\operatorname{Tr}(\boldsymbol{U})}{\alpha}+\sum_{n=1}^{N}\left(t_{n} p_{\text {on }}+\left(1-t_{n}\right) p_{\text {off }}\right), \\
& \text { s.t }(C 1): \operatorname{Tr}\left(\boldsymbol{U} \boldsymbol{F}_{n}\right) \leq t_{n} p_{D A}, \forall n \in N,(C 2),(C 3):(16), \\
& (C 4):(17), \forall k \in K,(C 5):\left[\begin{array}{rr}
\boldsymbol{U} & \boldsymbol{u} \\
\boldsymbol{u}^{T} & 1
\end{array}\right] \succeq 0,(C 6): \boldsymbol{U} \succeq 0 .
\end{aligned}
$$

where $F_{n}=\operatorname{diag}(\underbrace{0 \ldots 0}_{n-1}, 1, \underbrace{0, \ldots 0}_{N-n})$ is an auxiliary diagonal matrix whose elements are zero except the $n$-th element, $\forall n \in N$. The problem in (18) is still non-convex due to the binary variables in $(C 2): t_{n}=\{0,1\}, \forall n \in N$, which can be relaxed to the following two constraints [62]

$$
(C 2 a): t_{n}=[0,1],(C 2 b): \sum_{n=1}^{N} t_{n}-\sum_{n=1}^{N} t_{n}^{2} \leq 0, \forall n \in N,
$$

where $(C 2 a)$ is the relaxed version of the original constraint $(C 2)$, and $(C 2 b)$ confines the value of $t_{n}, \forall n \in N$, close to 0 or 1 . Hence, satisfying both constraints $(C 2 a)$ and $(C 2 b)$ equals to satisfying the original constraint $(C 2)$. The introduced constraint $(C 2 a)$ is simply a linear constraint, and the difficulty lies in handling $(C 2 b)$. Introducing a penalty factor $\varphi$, typically of large value, and moving $(C 2 b)$ into the objective function, the objective becomes into $\frac{\operatorname{Tr}(\boldsymbol{U})}{\alpha}+$ $\sum_{n=1}^{N}\left(t_{n} p_{\text {on }}+\left(1-t_{n}\right) p_{\text {off }}\right)+\varphi\left(\sum_{n=1}^{N} t_{n}-\sum_{n=1}^{N^{\alpha}} t_{n}^{2}\right)$. The formulated objective function shares the same optimal design policy and result with the original one [62]. It is because with a large value of the penalty factor $\varphi$, the term $\varphi\left(\sum_{n=1}^{N} t_{n}-\sum_{n=1}^{N} t_{n}^{2}\right)$ in the objective function naturally pushes the value of $t_{n}, \forall n \in N$, close to 0 or 1 , which exactly equals to the original constraint $(C 2)$. The last difficulty lies in the non-convex term $\varphi\left(\sum_{n=1}^{N} t_{n}-\sum_{n=1}^{N} t_{n}^{2}\right)$ in the objective function. It is observed that $\sum_{n=1}^{N} t_{n}-\sum_{n=1}^{N} t_{n}^{2}$ is the difference of two convex functions w.r.t the variable $t_{n}$, and thus can be handled by successive convex approximation such that $\sum_{n=1}^{N} t_{n}-\sum_{n=1}^{N} t_{n}^{2} \leq \sum_{n=1}^{N} t_{n}-\sum_{n=1}^{N}\left(t_{n}^{(i)}\right)^{2}-$
$2 \sum_{n=1}^{N} t_{n}^{(i)}\left(t_{n}-t_{n}^{(i)}\right)$, where $t_{n}^{(i)}$ denotes the value of $t_{n}$ at the $i$-th iteration [61] and is updated in an iterative way to tighten the approximation. Therefore, the optimization becomes into

$$
\begin{aligned}
& P 1(d)(\mathrm{DA}-\text { imperfect - prob) : } \\
& \underset{\boldsymbol{u}, \boldsymbol{t}}{\operatorname{argmin}} \frac{\operatorname{Tr}(\boldsymbol{U})}{\alpha}+\sum_{n=1}^{N}\left(t_{n} p_{\text {on }}+\left(1-t_{n}\right) p_{\text {off }}\right)+ \\
& \quad \varphi\left(\sum_{n=1}^{N} t_{n}-\sum_{n=1}^{N}\left(t_{n}^{(i)}\right)^{2}-2 \sum_{n=1}^{N} t_{n}^{(i)}\left(t_{n}-t_{n}^{(i)}\right)\right), \\
& \text { s.t }(C 1),(C 2 a): t_{n}=[0,1], \forall n \in N,(C 3)-(C 6) .
\end{aligned}
$$

Now P1(d) (DA-imperfect-prob) is a standard semi-definite programming (SDP) problem, which can be readily solved by CVX. The loss of optimality only comes from relaxing the term $\varphi\left(\sum_{n=1}^{N} t_{n}-\sum_{n=1}^{N} t_{n}^{2}\right)$ by successive convex approximation. Since the term $\sum_{n=1}^{N} t_{n}-\sum_{n=1}^{N} t_{n}^{2}$ is the difference of two convex functions w.r.t the variables $t_{n}, \forall n \in N$, it can be relaxed into the difference of a convex function and a linear function $\sum_{n=1}^{N} t_{n}-\sum_{n=1}^{N}\left(t_{n}^{(i)}\right)^{2}-2 \sum_{n=1}^{N} t_{n}^{(i)}\left(t_{n}-t_{n}^{(i)}\right)$, which serves as a strict upper bound of the original term. By updating the value of $t_{n}^{(i)}, \forall n \in N$, in each iteration, nearoptimality is achieved benefiting from the convexity of the re-formulated problem P1(d) (Eq. (20)). Moreover, since the successive convex approximation serves as the upper bound of the original problem, the optimization problem $\mathrm{P} 1(\mathrm{~d})$ is iteratively minimized and also lower bounded by the IR's SINR and physical layer security constraints. Hence, the nearoptimality and convergence of the algorithm are confirmed [62] [63]. Finally, the solver for P1(d) is summarized as follows.

Remark 1: Now we revisit the inequalities in (11), and observe that the first inequality in (11) actually contains two inequalities (also applicable for the second inequality in (11))

$$
\begin{aligned}
& -\overline{\boldsymbol{a}}_{d, 1}^{T}\left[\boldsymbol{u}_{R}^{T}, \boldsymbol{u}_{I}^{T}\right]^{T} \geq \sigma_{n} \sqrt{\bar{\Gamma}_{d}} \tan \theta, \text { and } \\
& \left\|\Theta_{d, 1}^{\frac{1}{2}}\left[\boldsymbol{u}_{R}^{T}, \boldsymbol{u}_{I}^{T}\right]^{T}\right\|_{2} \leq \frac{-\overline{\boldsymbol{a}}_{d, 1}^{T}\left[\boldsymbol{u}_{R}^{T}, \boldsymbol{u}_{I}^{T}\right]^{T}-\sigma_{n} \sqrt{\bar{\Gamma}_{d}} \tan \theta}{\Phi^{-1}\left(\eta_{d}\right)},
\end{aligned}
$$




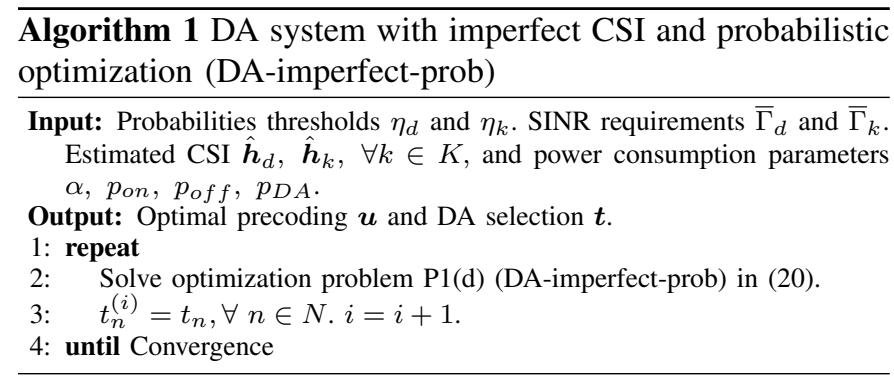

where the first inequality of (21) suggests that the magnitude of $\boldsymbol{u}$ should be large enough while the second inequality of (21) suggests that the magnitude of $\boldsymbol{u}$ should be smaller enough to make (21) holds. Hence, given a high channel estimation error (the norm of matrix $\Theta_{d, 1}^{\frac{1}{2}}$ is large), the value of $\eta_{d}$ or $\bar{\Gamma}_{d}$ needs to be properly reduced to make the optimization feasible. Revisiting the constraint for the $k$-th Eve in (14), we get $\left\|\Theta_{k, 1}^{\frac{1}{2}}\left[\boldsymbol{u}_{R}^{T}, \boldsymbol{u}_{I}^{T}\right]^{T}\right\|_{2} \leq \frac{\sigma_{n} \sqrt{\bar{\Gamma}_{k}} \tan \theta-\overline{\boldsymbol{a}}_{k, 1}^{T}\left[\boldsymbol{u}_{R}^{T}, \boldsymbol{u}_{I}^{T}\right]^{T}}{\Phi^{-1}\left(\eta_{k}\right)}$, and $\overline{\boldsymbol{a}}_{k, 1}^{T}\left[\boldsymbol{u}_{R}^{T}, \boldsymbol{u}_{I}^{T}\right]^{T} \leq \sigma_{n} \sqrt{\bar{\Gamma}_{k}} \tan \theta$, where only upper bounds on the magnitude of $\boldsymbol{u}$ are imposed. As a result, the constraint itself always confines a feasible region.

\section{B. Deterministic Robust Optimization}

In the previous subsection, we have solved the problem in a probabilistic manner, where the IR's QoS requirement and the physical layer security against the Eves are issued by the chance constrained formulations. In this subsection, we handle the CSI uncertainties from a deterministic manner, where the IR's QoS requirement and the physical layer security towards the Eves are always guaranteed with the infinite CSI uncertainties. A so-called DA-imperfect-det optimization problem is firstly formulated by P2(a) (Eq. 22), and then the subsequent optimization form P2(b) (Eq. 28) is obtained by applying S-procedure to handle non-convex constraints (C9) and (C10). At last, the final version in P2(c) (Eq. 29) is obtained by relaxing the binary variables in antenna selection.

1) Problem Formulation: Define $\boldsymbol{\Delta}$ as the channel estimation uncertainties set, which contains all the possible CSI uncertainties and specifies an ellipsoidal uncertainty region for the estimated CSI [65]. To process the power minimization problem in terms of deterministic robust optimization, the formulation is given as

$$
\begin{aligned}
& P 2(a)(\mathrm{DA}-\text { imperfect }- \text { det) : } \\
& \underset{\boldsymbol{w}, \boldsymbol{z}, \boldsymbol{t}}{\operatorname{argmin}} \frac{\| \boldsymbol{w}+\left.\boldsymbol{z} e^{-j \phi_{d}}\right|^{2}}{\alpha}+\sum_{n=1}^{N}\left(t_{n} p_{\text {on }}+\left(1-t_{n}\right) p_{o f f}\right), \\
& \text { s.t }(C 7): 0 \leq\left|w_{n}+z_{n} e^{-j \phi_{d}}\right|^{2} \leq t_{n} p_{D A}, \forall n \in N, \\
& \quad(C 8): t_{n}=\{0,1\}, \forall n \in N,(C 9): \min _{\boldsymbol{e}_{\boldsymbol{d}} \in \boldsymbol{\Delta}} \Gamma_{d} \geq \bar{\Gamma}_{d}, \\
& \quad(C 10): \max _{\boldsymbol{e}_{\boldsymbol{k}} \in \boldsymbol{\Delta}} \Gamma_{k} \leq \bar{\Gamma}_{k}, k \in K,
\end{aligned}
$$

where $(C 9)$ and $(C 10)$ indicate deterministic SINR requirement for the IR and physical layer security constraints against Eves, such that the IR's worst-case SINR and Eves' best-case SINR as per the CSI error distribution obey the respective thresholds $\bar{\Gamma}_{d}$ and $\bar{\Gamma}_{k}, \forall k \in K$.
2) Optimization Solution: In line with the analysis in previous subsection and defining $\boldsymbol{w}+\boldsymbol{z} e^{-j \phi_{d}}=\boldsymbol{u}$, constraint $(C 9)$ is equivalent to the following two inequalities in (23), as shown on the top of next page, where $\varrho_{d, 1}=\sigma_{n} \sqrt{\bar{\Gamma}_{d}} \tan \theta+$ $\hat{\boldsymbol{h}}_{I, d}^{T} \boldsymbol{u}_{R}+\hat{\boldsymbol{h}}_{R, d}^{T} \boldsymbol{u}_{I}-\hat{\boldsymbol{h}}_{R, d}^{T} \boldsymbol{u}_{R} \tan \theta+\hat{\boldsymbol{h}}_{I, d}^{T} \boldsymbol{u}_{I} \tan \theta, \varrho_{d, 2}=$ $\sigma_{n} \sqrt{\bar{\Gamma}_{d}} \tan \theta-\hat{\boldsymbol{h}}_{I, d}^{T} \boldsymbol{u}_{R}-\hat{\boldsymbol{h}}_{R, d}^{T} \boldsymbol{u}_{I}-\hat{\boldsymbol{h}}_{R, d}^{T} \boldsymbol{u}_{R} \tan \theta+\hat{\boldsymbol{h}}_{I, d}^{T} \boldsymbol{u}_{I} \tan \theta$.

We now handle the first inequality of (23). To handle the infinite CSI uncertainties, we transform it into a LMI using the following Lemma 1:

Lemma 1 (S-Procedure [64]): Let a function $f_{m}(\boldsymbol{x}), m \in$ $\{1,2\}$, be defined as $f_{m}(\boldsymbol{x})=\boldsymbol{x}^{H} \boldsymbol{A}_{m} \boldsymbol{x}+2 \Re\left\{\boldsymbol{b}_{m}^{H} \boldsymbol{x}\right\}+c_{m}$, where $\boldsymbol{A}_{m} \in \mathbb{H}^{N \times N}, \boldsymbol{b}_{m} \in \mathbb{C}^{N \times 1}$ and $c_{m} \in \mathbb{R}$. The implication $f_{1}(\boldsymbol{x}) \leq 0 \Rightarrow f_{2}(\boldsymbol{x}) \leq 0$ holds if and only if there exists an $\lambda \geq 0$ such that $\lambda\left[\begin{array}{ll}\boldsymbol{A}_{1}, & \boldsymbol{b}_{1} \\ \boldsymbol{b}_{1}^{H}, & c_{1}\end{array}\right]-\left[\begin{array}{ll}\boldsymbol{A}_{2}, & \boldsymbol{b}_{2} \\ \boldsymbol{b}_{2}^{H}, & c_{2}\end{array}\right] \succeq \mathbf{0 .}$

By exploiting the structure of the first inequality in (23), we need to construct an inequality such that $\left[\sqrt{\left(\boldsymbol{e}_{I, d}-\boldsymbol{e}_{R, d} \tan \theta\right)^{T}}, \quad \sqrt{\left(\boldsymbol{e}_{R, d}+\boldsymbol{e}_{I, d} \tan \theta\right)^{T}}\right] \quad \boldsymbol{I}_{2 N}$ $\left[\sqrt{\left(\boldsymbol{e}_{I, d}-\boldsymbol{e}_{R, d} \tan \theta\right)^{T}}, \sqrt{\left(\boldsymbol{e}_{R, d}+\boldsymbol{e}_{I, d} \tan \theta\right)^{T}}\right]^{T}-\sigma_{d}^{U B} \leq 0$ to guarantee the first inequality in (23) always hold. We note that expanding the term yields a linear combination of the elements of $\boldsymbol{e}_{d}$. Since the 2-norm of the channel estimation error vector $\boldsymbol{e}_{d}$ is bounded by the uncertainty-bounded channel estimation error model, the linear combination of the elements of $e_{d}$ is also bounded [65]. Hence, without loss of generality, $\sigma_{d}^{U B}$ is assumed to serve as the upper bound of the constructed term. By applying S-procedure, the constructed inequality guarantees the first inequality in (23) hold if and only if there exists $\lambda_{d, 1} \geq 0$ such that the LMI constraint in (24) holds

$$
\left[\begin{array}{cc}
\lambda_{d, 1} \boldsymbol{I}_{2 N}-\operatorname{diag}\left(\boldsymbol{u}_{R}^{T}, \boldsymbol{u}_{I}^{T}\right), & \mathbf{0} \\
\mathbf{0}, & -\lambda_{d, 1} \sigma_{d}^{U B}-\varrho_{d, 1}
\end{array}\right] \succeq \mathbf{0},
$$

by which the uncertainties have been omitted. Now the first constraint in (23) containing infinite possibilities is transformed into a solvable LMI constraint in (24). Accordingly, the second inequality in (23) can be transformed into

$$
\left[\begin{array}{cc}
\lambda_{d, 2} \boldsymbol{I}_{2 N}-\operatorname{diag}\left(\boldsymbol{u}_{R}^{T}, \boldsymbol{u}_{I}^{T}\right), & \mathbf{0} \\
\mathbf{0}, & -\lambda_{d, 2} \sigma_{d}^{U B}-\varrho_{d, 2}
\end{array}\right] \succeq \mathbf{0} .
$$

Now the constraint $(C 9)$ containing infinite possibilities is transformed into two deterministic LMIs in (24) and (25), respectively. Now we handle the $k$-th Eve's constraint in $(C 10)$. According to the geometrical interpretation in Fig. 2, (C10) can be equivalently written by (26), as shown on the top of next page, where $\varrho_{k, 1}=\hat{\boldsymbol{h}}_{I, k}^{T} \boldsymbol{u}_{R}+\hat{\boldsymbol{h}}_{R, k}^{T} \boldsymbol{u}_{R} \tan \theta+$ $\hat{\boldsymbol{h}}_{R, k}^{T} \boldsymbol{u}_{I}-\hat{\boldsymbol{h}}_{I, k}^{T} \boldsymbol{u}_{I} \tan \theta-\sigma_{n} \sqrt{\bar{\Gamma}_{k}} \tan \theta$, and $\varrho_{k, 2}=-\hat{\boldsymbol{h}}_{I, k}^{T} \boldsymbol{u}_{R}+$ $\hat{\boldsymbol{h}}_{R, k}^{T} \boldsymbol{u}_{R} \tan \theta-\hat{\boldsymbol{h}}_{R, k}^{T} \boldsymbol{u}_{I}-\hat{\boldsymbol{h}}_{I, k}^{T} \boldsymbol{u}_{I} \tan \theta-\sigma_{n} \sqrt{\overline{\bar{\Gamma}}_{k}} \tan \theta$. Based on S-procedure introduced above, (26) can be transformed into

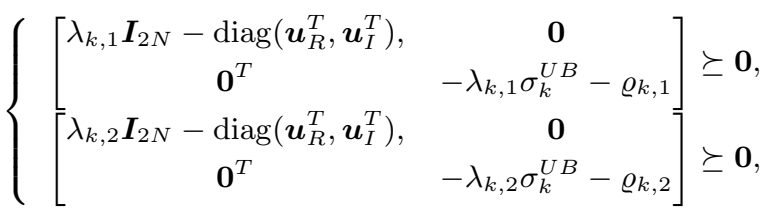




$$
\left\{\begin{array}{l}
\min _{\boldsymbol{e}_{\boldsymbol{d}} \in \boldsymbol{\Delta}}\left[\left(\boldsymbol{e}_{I, d}-\boldsymbol{e}_{R, d} \tan \theta\right)^{T},\left(\boldsymbol{e}_{R, d}+\boldsymbol{e}_{I, d} \tan \theta\right)^{T}\right]\left[\boldsymbol{u}_{R}^{T}, \boldsymbol{u}_{I}^{T}\right]^{T}+\varrho_{d, 1} \leq 0, \\
\min _{\boldsymbol{e}_{\boldsymbol{d}} \in \boldsymbol{\Delta}}\left[\left(-\boldsymbol{e}_{I, d}-\boldsymbol{e}_{R, d} \tan \theta\right)^{T},\left(-\boldsymbol{e}_{R, d}+\boldsymbol{e}_{I, d} \tan \theta\right)^{T}\right]\left[\boldsymbol{u}_{R}^{T}, \boldsymbol{u}_{I}^{T}\right]^{T}+\varrho_{d, 2} \leq 0,
\end{array}\right.
$$

$$
\left\{\begin{array}{l}
\max _{\boldsymbol{e}_{\boldsymbol{k}} \in \boldsymbol{\Delta}}\left[\left(\boldsymbol{e}_{I, k}+\boldsymbol{e}_{R, k} \tan \theta\right)^{T},\left(\boldsymbol{e}_{R, k}-\boldsymbol{e}_{I, k} \tan \theta\right)^{T}\right]\left[\boldsymbol{u}_{R}^{T}, \boldsymbol{u}_{I}^{T}\right]^{T}+\varrho_{k, 1} \leq 0, \\
\max _{\boldsymbol{e}_{\boldsymbol{k}} \in \boldsymbol{\Delta}}\left[\left(-\boldsymbol{e}_{I, k}+\boldsymbol{e}_{R, k} \tan \theta\right)^{T},\left(-\boldsymbol{e}_{R, k}-\boldsymbol{e}_{I, k} \tan \theta\right)^{T}\right]\left[\boldsymbol{u}_{R}^{T}, \boldsymbol{u}_{I}^{T}\right]^{T}+\varrho_{k, 2} \leq 0 .
\end{array}\right.
$$

where $\sigma_{k}^{U B}$ is similarly introduced to serve as an upper bound of the linear combination of the elements of $\boldsymbol{e}_{k}$. Now the nonconvex constraints $(C 9)$ and $(C 10)$ are replaced by equivalent LMI constraints. Defining $\boldsymbol{U}=\boldsymbol{u}^{H} \boldsymbol{u}$, the problem can be transformed as

$$
\begin{aligned}
& P 2(b)(\mathrm{DA}-\text { imperfect }- \text { det }): \\
& \underset{\boldsymbol{w}, \boldsymbol{z}, \boldsymbol{t}}{\operatorname{argmin}} \frac{\operatorname{Tr}(\boldsymbol{U})}{\alpha}+\sum_{n=1}^{N}\left(t_{n} p_{o n}+\left(1-t_{n}\right) p_{o f f}\right), \\
& \text { s.t }(C 7): \operatorname{Tr}\left(\boldsymbol{U} \boldsymbol{F}_{n}\right) \leq t_{n} p_{D A}, \forall n \in N,(C 9):(24) \text { and }(25), \\
& (C 8): t_{n}=\{0,1\}, \forall n \in N,(C 10):(27), \forall k \in K, \\
& (C 11):\left[\begin{array}{rr}
\boldsymbol{U} & \boldsymbol{u} \\
\boldsymbol{u}^{T} & 1
\end{array}\right] \succeq 0,(C 12): \boldsymbol{U} \succeq 0,(C 13): \lambda_{d, 1} \geq 0, \\
& (C 14): \lambda_{d, 2} \geq 0,(C 15): \lambda_{k, 1} \geq 0,(C 16): \lambda_{k, 2} \geq 0, \forall k \in K .
\end{aligned}
$$

To handle $(C 8)$, we transform it into two equivalent constraints $(C 8 a): t_{n}=[0,1],(C 8 b): \sum_{n=1}^{N} t_{n}-\sum_{n=1}^{N} t_{n}^{2} \leq$ $0, \forall n \in N$. Then we introduce a penalty factor $\varphi$, typically of large value, and move $(C 8 b)$ into the objective function. By applying successive convex approximation for the non-convex term $\varphi\left(\sum_{n=1}^{N} t_{n}-\sum_{n=1}^{N} t_{n}^{2}\right)$, the problem becomes

$$
\begin{aligned}
P 2(c)(\mathrm{DA}-\text { imperfect }- \text { det }): & \underset{\boldsymbol{w}, \boldsymbol{z}, \boldsymbol{t}}{\operatorname{argmin}} \frac{\operatorname{Tr}(\boldsymbol{U})}{\alpha}+\sum_{n=1}^{N}\left(t_{n} p_{o n}+\left(1-t_{n}\right) p_{o f f}\right)+ \\
& \varphi\left(\sum_{n=1}^{N} t_{n}-\sum_{n=1}^{N}\left(t_{n}^{(i)}\right)^{2}-2 \sum_{n=1}^{N} t_{n}^{(i)}\left(t_{n}-t_{n}^{(i)}\right)\right),
\end{aligned}
$$

$$
\text { s.t }(C 7),(C 8): t_{n} \in[0,1],(C 9)-(C 16),
$$

which is ready to be solved as a standard SDP problem. The solver for the deterministic robust optimization problem is summarized by Algorithm 2, and its near-optimality and convergence are similar to that of the Algorithm 1.

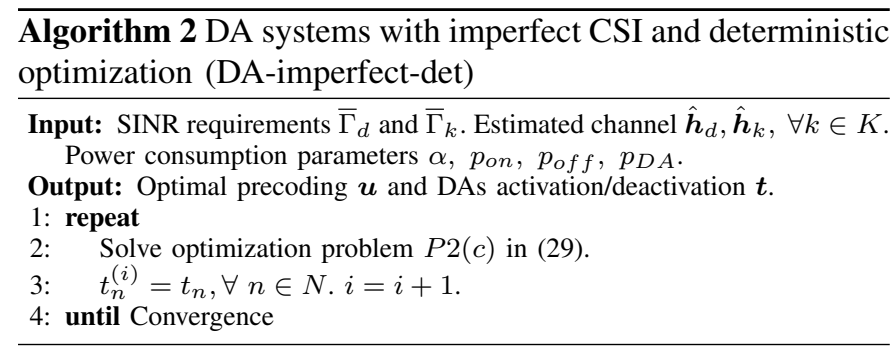

Remark 2: By the deterministic manner, the SINR requirements need to be always satisfied at the cost of high transmission power. In contrast, by the probabilistic manner, the statistical SINR constraints allow proper violations, while obeying the pre-set outage thresholds. Moreover, when processing robust optimization deterministically, more intermediate variables are introduced by applying S-procedure. As a result, the deterministic optimization generally leads to high power consumption and complexity.

\section{Power EfFicient DA Selection And Precoding Without EVES' CSI}

In a number of practical scenarios, it is impossible to obtain the Eves' CSI information. In this section, we investigate security issue when imperfect CSI is only available for the IR while the Eves' CSI is completely unknown. Again, we solve the problem in probabilistic and deterministic manners.

\section{A. Probabilistic Robust Optimization}

To solve the problem in a probabilistic manner, the channel estimation error is modeled by a standard normal distributed variable, as presented in subsection III-A. A so-called DAunknown-prob optimization problem is firstly formulated by P3(a) (Eq. 30), and then the subsequent optimization form P3(b) (Eq. 31) is obtained by handling the non-convex constraint (C19). At last, the final version s obtained in P3(c) (Eq. 32) by relaxing the binary variables in antenna selection. Since the IR' CSI can be imperfectly obtained, we can still guarantee the IR' SINR requirement by a probabilistic constraint. However, to address physical layer security in absence of any Eves' CSI, we can only set a minimum power level of AN.

1) Problem Formulation: The problem is formulated as

$$
\begin{aligned}
& P 3(a)(\mathrm{DA}-\text { unknown }- \text { prob) : } \\
& \underset{\boldsymbol{w}, \boldsymbol{z}, \boldsymbol{t}}{\operatorname{argmin}} \frac{\left\|\boldsymbol{w}+\boldsymbol{z} e^{-j \phi_{d}}\right\|^{2}}{\alpha}+\sum_{n=1}^{N}\left(t_{n} p_{o n}+\left(1-t_{n}\right) p_{o f f}\right), \\
& \text { s.t }(C 17): 0 \leq\left|w_{n}+z_{n} e^{-j \phi_{d}}\right|^{2} \leq t_{n} p_{D A}, \forall n \in N, \\
& (C 18): t_{n}=\{0,1\}, \forall n \in N, \\
& (C 19): \operatorname{Pr}\left\{\Gamma_{d} \geq \bar{\Gamma}_{d}\right\} \geq \eta_{d},(C 20):\|\boldsymbol{z}\|^{2} \geq P_{A N},
\end{aligned}
$$

where constraint $(C 20)$ is imposed to guarantee the minimum power level of AN and is of importance when the Eves' CSI is unknown [43]. The essence of constraint (C20) is further summarized in Appendix I for brevity.

2) Optimization Solution: To solve the problem, we transform the probabilistic constraint $(C 19)$ into equivalent LMI, as shown by (16). Define $\boldsymbol{u}=\boldsymbol{w}+\boldsymbol{z} e^{-j \phi_{d}}, \boldsymbol{U}=\boldsymbol{u}^{H} \boldsymbol{u}$ and $\boldsymbol{Z}=\boldsymbol{z}^{H} \boldsymbol{z}$. The problem is given by 


$$
\begin{aligned}
& P 3(b)(\mathrm{DA}-\text { unknown }- \text { prob }): \\
& \underset{\boldsymbol{w}, \boldsymbol{z}, \boldsymbol{t}}{\operatorname{argmin}} \frac{\operatorname{Tr}(\boldsymbol{U})}{\alpha}+\sum_{n=1}^{N}\left(t_{n} p_{o n}+\left(1-t_{n}\right) p_{o f f}\right) \\
& \text { s.t }(C 17): \operatorname{Tr}\left(\boldsymbol{U} \boldsymbol{F}_{n}\right) \leq t_{n} p_{D A}, \forall n \in N,(C 18) .(C 19):(16), \\
& (C 20): \operatorname{Tr}(\boldsymbol{Z}) \geq p_{A N},(C 21): \operatorname{Tr}\left(\boldsymbol{Z} \boldsymbol{F}_{n}\right) \leq \operatorname{Tr}\left(\boldsymbol{U} \boldsymbol{F}_{n}\right), \\
& (C 22): \boldsymbol{w}+\boldsymbol{z} e^{-j \phi_{d}}=\boldsymbol{u},(C 23):\left[\begin{array}{cc}
\boldsymbol{U} & \boldsymbol{u} \\
\boldsymbol{u}^{T} & 1
\end{array}\right] \succeq 0, \\
& (C 24):\left[\begin{array}{cc}
\boldsymbol{Z} & \boldsymbol{z} \\
\boldsymbol{z}^{T} & 1
\end{array}\right] \succeq 0,(C 25): \boldsymbol{U} \succeq 0,(C 26): \boldsymbol{Z} \succeq 0 .
\end{aligned}
$$

where constraint $(C 21)$ is imposed to guarantee that the AN generated on each DA is lower than the overall beamformer weight. For the binary variables involved constraint $(C 18)$, we again transform it into two equivalent constraints $(C 18 a)$ : $t_{n}=[0,1],(C 18 b): \sum_{n=1}^{N} t_{n}-\sum_{n=1}^{N} t_{n}^{2} \leq 0, \forall n \in N$, Then we introduce a penalty factor $\varphi$, typically of large value, and move $(C 18 b)$ into the objective function. By applying successive convex approximation for the non-convex term $\varphi\left(\sum_{n=1}^{N} t_{n}-\sum_{n=1}^{N} t_{n}^{2}\right)$, the formulated problem becomes

$$
\begin{aligned}
& P 3(c)(\mathrm{DA}-\text { unknown - prob }): \\
& \underset{\boldsymbol{w}, \boldsymbol{z}, \boldsymbol{t}}{\operatorname{argmin}} \frac{\operatorname{Tr}(\boldsymbol{U})}{\alpha}+\sum_{n=1}^{N}\left(t_{n} p_{\text {on }}+\left(1-t_{n}\right) p_{\text {off }}\right)+ \\
& \quad \varphi\left(\sum_{n=1}^{N} t_{n}-\sum_{n=1}^{N}\left(t_{n}^{(i)}\right)^{2}-2 \sum_{n=1}^{N} t_{n}^{(i)}\left(t_{n}-t_{n}^{(i)}\right)\right), \\
& \text { s.t }(C 17),(C 18 a): t_{n}=[0,1], \forall n \in N,(C 9)-(C 26),
\end{aligned}
$$

which is ready to solve as a standard SDP problem. The solver is summarized in Algorithm 3, and its near-optimality and convergence are similar to that of the Algorithm 1.

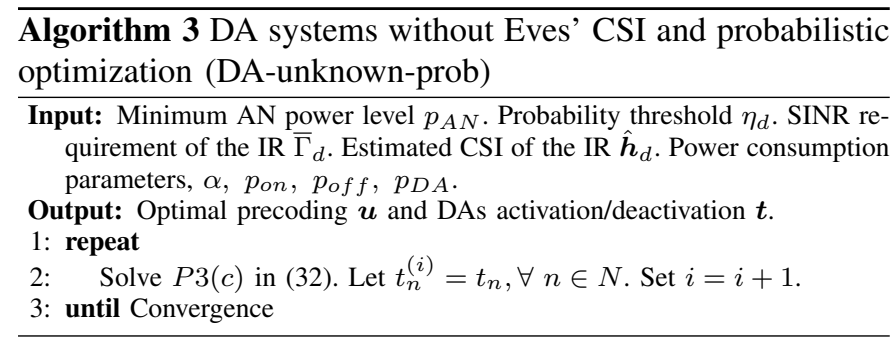

\section{B. Deterministic Robust Optimization}

Now we present the power efficient design in deterministic manner, for the case when the Eves' CSI is unknown. To guarantee the IR's SINR in a deterministic manner, we again define $\boldsymbol{\Delta}$ as the channel estimation uncertainties set, which contains all the possible CSI uncertainties and specifies an ellipsoidal uncertainty region for the estimated CSI [65]. A so-called DA-unknown-det optimization problem is firstly formulated by P4(a) (Eq. 33). After processing the non-convex constraint (C29) and relaxing the binary variables in antenna selection, its final version is demonstrated by $\mathrm{P} 4(\mathrm{~b})$ in (34).
1) Problem Formulation: As discussed above, the IR's SINR needs to be satisfied with all the CSI uncertainties. Again, we set a minimum power level of AN to address physical layer security issue. The problem is formulated as

$$
\begin{aligned}
& P 4(a)(\mathrm{DA}-\text { unknown }-\mathrm{det}): \\
& \underset{\boldsymbol{w}, \boldsymbol{z}, \boldsymbol{t}}{\operatorname{argmin}} \frac{\left\|\boldsymbol{w}+\boldsymbol{z} e^{-j \phi_{d}}\right\|^{2}}{\alpha}+\sum_{n=1}^{N}\left(t_{n} p_{o n}+\left(1-t_{n}\right) p_{o f f}\right), \\
& \text { s.t }(C 27): 0 \leq\left|w_{n}+z_{n} e^{-j \phi_{d}}\right|^{2} \leq t_{n} p_{D A}, \forall n \in N, \\
& \quad(C 28): t_{n}=\{0,1\}, \forall n \in N, \\
& \quad(C 29): \min _{\boldsymbol{e}_{\boldsymbol{d}} \in \boldsymbol{\Delta}} \Gamma_{d} \geq \bar{\Gamma}_{d},(C 30):\|\boldsymbol{z}\|^{2} \geq P_{A N},
\end{aligned}
$$

where $(C 29)$ denotes that the minimum possible SINR of the IR should be higher than the required $\bar{\Gamma}_{d}$ with all the CSI uncertainties.

2) Optimization Solution: By applying S-procedure, we transform the constraint $(C 29)$ that contains infinity probabilities into equivalent LMIs, as shown by Eqs. (24) and (25). Then we handle the binary variables involved constraint $(C 28)$ by success convex approximation. Define $\boldsymbol{u}=\boldsymbol{w}+\boldsymbol{z} e^{-j \phi_{d}}$, $\boldsymbol{U}=\boldsymbol{u}^{H} \boldsymbol{u}$ and $\boldsymbol{Z}=\boldsymbol{z}^{H} \boldsymbol{z}$. The transformed problem becomes

$$
\begin{aligned}
& P 4(b)(\text { DA - unknown - det) : } \\
& \underset{\boldsymbol{w}, \boldsymbol{z}, \boldsymbol{t}}{\operatorname{argmax}} \frac{\operatorname{Tr}(\boldsymbol{U})}{\alpha}+\sum_{n=1}^{N}\left(t_{n} p_{\text {on }}+\left(1-t_{n}\right) p_{\text {off }}\right)+ \\
& \varphi\left(\sum_{n=1}^{N} t_{n}-\sum_{n=1}^{N}\left(t_{n}^{(i)}\right)^{2}-2 \sum_{n=1}^{N} t_{n}^{(i)}\left(t_{n}-t_{n}^{(i)}\right)\right), \\
& \text { s.t }(C 27): \operatorname{Tr}\left(\boldsymbol{U} \boldsymbol{F}_{n}\right) \leq t_{n} p_{D A}, \forall n \in N \text {, } \\
& (C 28): t_{n}=\{0,1\}, \forall n \in N,(C 29):(24) \text { and (25), } \\
& (C 30): \operatorname{Tr}(\boldsymbol{Z}) \geq p_{A N},(C 31): \operatorname{Tr}\left(\boldsymbol{Z} \boldsymbol{F}_{n}\right) \leq \operatorname{Tr}\left(\boldsymbol{U} \boldsymbol{F}_{n}\right) \text {, } \\
& (C 32): \boldsymbol{w}+\boldsymbol{z} e^{-j \phi_{d}}=\boldsymbol{u},(C 33):\left[\begin{array}{cc}
\boldsymbol{U} & \boldsymbol{u} \\
\boldsymbol{u}^{T} & 1
\end{array}\right] \succeq 0, \\
& (C 34):\left[\begin{array}{ll}
\boldsymbol{Z} & \boldsymbol{z} \\
\boldsymbol{z}^{T} & 1
\end{array}\right] \succeq 0,(C 35): \boldsymbol{U} \succeq 0,(C 36): \boldsymbol{Z} \succeq 0, \\
& (C 37): \lambda_{d, 1} \geq 0,(C 38): \lambda_{d, 2} \geq 0,
\end{aligned}
$$

which is ready to be solved as a standard SDP problem, and the solver is given by Algorithm 4. Its near-optimality and convergence are similar to that of the Algorithm 1.

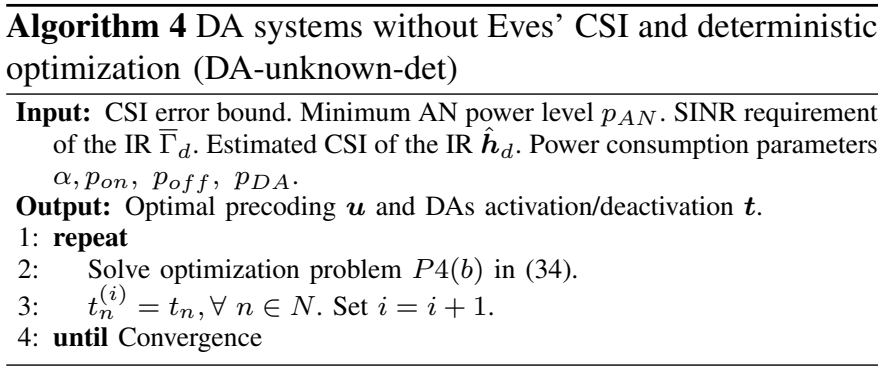

\section{COMPLEXITY ANALYSIS AND CONSTRUCTIVE AN DESIGN FOR GENERIC MODULATION SCHEMES}

In this section we analytically examine the computational complexity of the proposed algorithms, and also discuss the applicability of constructive AN on other constellation schemes. 


\section{A. Complexity Analysis}

We first examine the computational complexity of the proposed algorithms, and benchmark them against the related schemes in [3] [21] [22] [24] [38]. For a fair comparison, the parameters in different algorithms have been modified to be consistent (the same number of users and antennas). It can be seen that the proposed algorithms run in an iterative manner, and we have several LMI and linear inequalities to handle in each iteration. For the interior-point methods based solver, the overall complexity can be given as $\ln \left(\frac{1}{\epsilon}\right) l_{i} \sqrt{c_{b}}\left(c_{f}+c_{g}\right)$ [66]. Specifically, $\ln \left(\frac{1}{\epsilon}\right)$ relates to the accuracy setup. $l_{i}$ represents the number of iterations for updating DA selection vector. $\sqrt{c_{b}}$ represents the barrier parameter measuring the geometric complexity of the conic constraints. $c_{f}$ and $c_{g}$ represent the complexities cost on forming and factorization of $n \times n$ matrix of the linear system ${ }^{3}$.

Finally, the overall complexities of the proposed algorithms are summarized in TABLE I. It is observed that the proposed algorithms have polynomial time computational complexity, and only $l_{\text {ite }}$ iterations for updating DA section vector is required. The value of $l_{i t e}$ is further demonstrated in Fig.10. Alternatively, by an exhaustive search, there are $2^{N}$ possibilities for antenna selection, which imposes extremely high computational complexity, especially given a large number of antennas. Given the above discussion, it is worth highlighting that, the proposed algorithms strike a favorable tradeoff between system performance and complexity. Moreover, compared to the probabilistic robust optimization, the deterministic optimization has $2+2 K$ more linear inequalities in the first scenario and 2 more linear inequalities in the second scenario. However, the additional linear inequalities have minimal impacts on the complexities as they only linearly increase with the number of devices.

\section{B. Constructive AN Design for Other Constellation Schemes}

The principle of constructive AN is also applicable for other constellation schemes, with a minor revision on (2). For illustration purpose, we provide a schematic representation for 16-QAM constellation points in Fig. 3. To guarantee constructive AN for the constellation points, we exploit the specific detection regions for each group of constellation points separately as detailed below.

For the group of constellation points in the box labelled 1: since they are all surrounded by the decision boundaries, the constraints should guarantee that the received signals achieve the exact constellation point so as not to exceed the decision boundaries. Hence, the constraints are

\footnotetext{
${ }^{3}$ Assume that the problem is subjected to P LMI and (M-P) second order cone (SOC) constraints, and the overall complexity consists of two parts: iteration complexity and per-iteration computation cost. (i) The iteration complexity is calculated as $\ln (1 / \epsilon) \sqrt{c_{b}}$, where $\ln (1 / \epsilon)$ relates to the accuracy setup, $c_{b}=\sum_{p=1}^{P} k_{p}+2(M-P)$ represents the barrier parameter measuring the geometric complexity of the conic constraints, and $k_{p}$ represents the size of the p-th LMI constraint. (ii) On the other hand, the per-iteration computation is further dominated by: a) the formation of the $n \times n$ coefficient matrix of the optimization problem, which is calculated as $c_{f}=n \sum_{p=1}^{P} k_{p}^{3}+n^{2} \sum_{p=1}^{P} k_{p}^{2}+n \sum_{p=P+1}^{m} k_{p}^{2}$. The first two terms come from the $\mathrm{P}$ LMI constraints while the third term comes from M-P second order cone constraints. b) the factorization of the coefficient matrix, which is calculated as $c_{g}=n^{3}$ (Eq. (18), [66]).
}

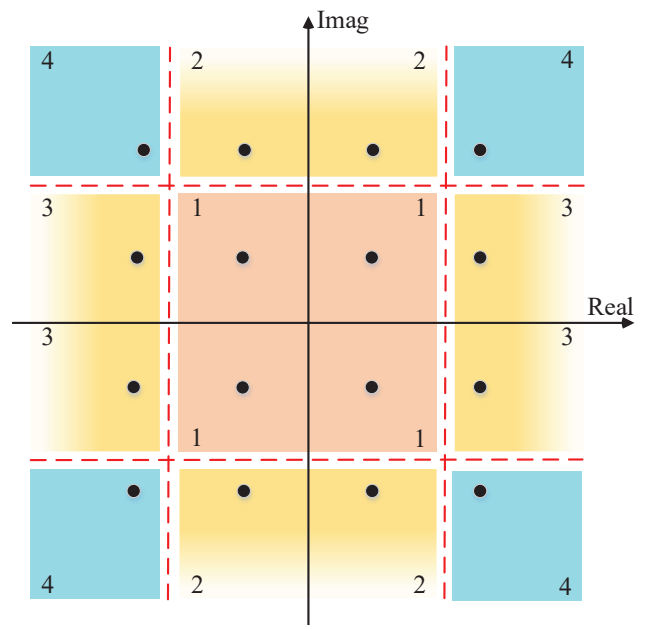

Fig. 3. Schematic representation of 16-QAM constellation points.

$$
\begin{aligned}
& \Re\left\{\boldsymbol{h}_{d}\left(\boldsymbol{w}+\boldsymbol{z} e^{-j \phi_{d}}\right)\right\}=\sqrt{\bar{\Gamma}_{d}} \sigma_{n} \Re\left\{x_{d}\right\}, \\
& \text { and } \Im\left\{\boldsymbol{h}_{d}\left(\boldsymbol{w}+\boldsymbol{z} e^{-j \phi_{d}}\right)\right\}=\sqrt{\bar{\Gamma}_{d}} \sigma_{n} \Im\left\{x_{d}\right\} .
\end{aligned}
$$

For the group of constellation points labelled 2: the constraints should guarantee that the received signals fall in the detection region away from the decision boundaries, which is the imaginary axis. The constraints are

$$
\begin{aligned}
& \Re\left\{\boldsymbol{h}_{d}\left(\boldsymbol{w}+\boldsymbol{z} e^{-j \phi_{d}}\right)\right\}=\sqrt{\bar{\Gamma}_{d}} \sigma_{n} \Re\left\{x_{d}\right\}, \\
& \text { and } \Im\left\{\boldsymbol{h}_{d}\left(\boldsymbol{w}+\boldsymbol{z} e^{-j \phi_{d}}\right)\right\} \geq(\text { or } \leq) \sqrt{\bar{\Gamma}_{d}} \sigma_{n} \Im\left\{x_{d}\right\} .
\end{aligned}
$$

For the group of constellation points labelled 3: the constraints should guarantee that the received signals fall in the detection region away from the decision boundaries, which is the real axis. The constraints are

$$
\begin{aligned}
& \Re\left\{\boldsymbol{h}_{d}\left(\boldsymbol{w}+\boldsymbol{z} e^{-j \phi_{d}}\right)\right\} \geq(\text { or } \leq) \sqrt{\bar{\Gamma}_{d}} \sigma_{n} \Re\left\{x_{d}\right\}, \\
& \text { and } \Im\left\{\boldsymbol{h}_{d}\left(\boldsymbol{w}+\boldsymbol{z} e^{-j \phi_{d}}\right)\right\}=\sqrt{\bar{\Gamma}_{d}} \sigma_{n} \Im\left\{x_{d}\right\} .
\end{aligned}
$$

For the group of constellation points labelled 4: the constraints should guarantee that the received signals fall in the detection region away from the decision boundaries. Here, the constellation points are not surrounded by the decision boundaries and that extend infinitely, and constraints are written as

$$
\begin{aligned}
& \Re\left\{\boldsymbol{h}_{d}\left(\boldsymbol{w}+\boldsymbol{z} e^{-j \phi_{d}}\right)\right\} \geq(\text { or } \leq) \sqrt{\bar{\Gamma}_{d}} \sigma_{n} \Re\left\{x_{d}\right\}, \\
& \text { and } \Im\left\{\boldsymbol{h}_{d}\left(\boldsymbol{w}+\boldsymbol{z} e^{-j \phi_{d}}\right)\right\} \geq(\text { or } \leq) \sqrt{\bar{\Gamma}_{d}} \sigma_{n} \Im\left\{x_{d}\right\} .
\end{aligned}
$$

\section{Simulation Results}

We present the simulated performance in this section. The central frequency is set to $2 \mathrm{GHz}$ with $1 \mathrm{MHz}$ bandwidth. The AWGN power spectral density is $-174 \mathrm{dBm} / \mathrm{Hz}$. A $100 \times 100$ $\mathrm{m}^{2}$ square cell model is considered with $N=16$ DAs, where the multiple DAs are evenly fixed [4] [67] across the map, as 
TABLE II. Complexity analysis ${ }^{4}$, with accuracy factor $\epsilon$

\begin{tabular}{|c|c|}
\hline DA-imperfect-prob & $\begin{array}{c}C_{i p}=\ln \left(\frac{1}{\epsilon}\right) l_{i} \sqrt{(9 N+3)+2 K(2 N+1)}\left[n\left(3 N+N^{3}+(N+1)^{3}+(2+2 K)(2 N+1)^{3}\right)\right. \\
\left.+n^{2}\left(3 N+N^{2}+(N+1)^{2}+(2+2 K)(2 N+1)^{2}\right)+n^{3}\right]\end{array}$ \\
\hline DA-imperfect-det & $\begin{array}{c}C_{i d}=\ln \left(\frac{1}{\epsilon}\right) l_{i} \sqrt{9 N+4 K+5+4 K 2 N}\left[n\left(3 N+2+2 K+(2+2 K)(2 N+1)^{3}\right.\right. \\
\left.+(N+1)^{3}+N^{3}\right)+n^{2}\left(3 N+2+2 K+(2 K+2)(N+1)^{2}+(N+1)^{2}+N^{2}\right)+n^{3}\end{array}$ \\
\hline DA-unknown-prob & $\begin{array}{c}C_{u p}=\ln \left(\frac{1}{\epsilon}\right) l_{i} \sqrt{13 N+5}\left[n\left(5 N+2 N^{3}+1+2(N+1)^{3}+2(2 N+1)^{3}\right)\right. \\
\left.+n^{2}\left(5 N+1+2 N^{2}+2(N+1)^{2}+2(2 N+1)^{2}\right)+n^{3}\right]\end{array}$ \\
\hline DA-unknown-det & $\begin{aligned} C_{u d}= & \ln \left(\frac{1}{\epsilon}\right) l_{i} \sqrt{13 N+7}\left[n\left(5 N+3+2 N^{3}+2(N+1)^{3}+2(2 N+1)^{3}\right)\right. \\
& \left.+n^{2}\left(5 N+3+2 N^{2}+2(N+1)^{2}+2(2 N+1)^{2}\right)+n^{3}\right]\end{aligned}$ \\
\hline CA-no-AS [38] & $\ln \left(\frac{1}{\epsilon}\right) \sqrt{(4+4 K)}\left[n\left(8 N^{2}+8 K N^{2}\right)+n^{3}\right]$ \\
\hline DA-conv-AN [3] & $\begin{array}{l}\ln \left(\frac{1}{\epsilon}\right) l_{\text {ite }} \sqrt{2 N L_{1}}\left[\left(1+2 K+3 L_{1}\right)\left(2 N L_{1}\right)^{3}+\left(2 N L_{1}\right)^{2}\left(1+2 K+3 L_{1}\right)^{2}\right. \\
\left.\quad+\left(1+2 K+3 L_{1}\right)^{3}\right], \text { where } L_{1} \text { denotes the number of remote radio heads }\end{array}$ \\
\hline Power-min algorithm [22] & $\ln \left(\frac{1}{\epsilon}\right) l_{i t e} \sqrt{2+2 N}\left[(K+1)\left(n+n^{2}\right)+2 n N^{3}+2 n^{2} N^{2}+n^{3}\right]$ \\
\hline Secrecy rate-max algorithm [21] & $\ln \left(\frac{1}{\epsilon}\right) l_{i t e} \sqrt{4+2 N}\left[4\left(n+n^{2}\right)+2\left(n N^{3}+n^{2} N^{2}\right)+n^{3}\right]$ \\
\hline Untrusted-relay algorithm [24] & $\begin{array}{c}\ln \left(\frac{1}{\epsilon}\right) l_{\text {ite }} \sqrt{3 L_{2} N+L_{2}+N\left[L_{2}\left(n+n^{2}\right)+\left(3 L_{2}+1\right)\left(n N^{3}+n^{2} N^{2}\right)+n^{3}\right]} \\
\text { where } L_{2} \text { denotes the number of users }\end{array}$ \\
\hline
\end{tabular}

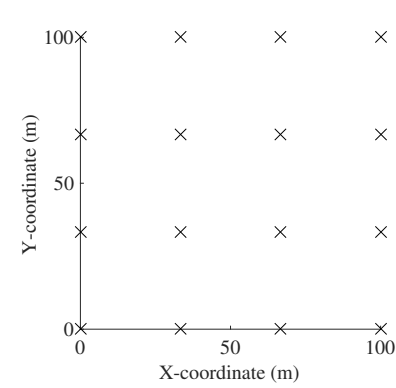

(a)

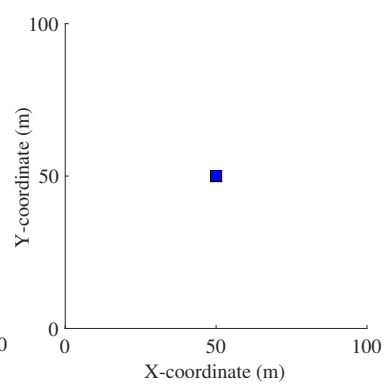

(b)

Fig. 4. (a) Antenna deployment in DA systems, where antennas are geographically distributed. (b) Antenna deployment in CA systems, where antennas are co-located in the map center.

shown in Fig. 4. The number of the Eves is $K=14$. The IR and Eves are randomly distributed across the map following uniform distribution [11]. To further highlight the advantages of the DA deployment, in Figs. 6, 9 and 10, subsets of the IR and Eves are partially placed at the coverage edge. DE of all PAs is set to $\alpha=40 \%$. Power consumption parameters are set to $p_{\text {on }}=500 \mathrm{~mW}, p_{\text {off }}=50 \mathrm{~mW}$, and $p_{D A}=1000$ $\mathrm{mW}$, respectively. CSI error variance (in subsections III-A and IV-A) and error upper bound (in subsections III-B and IV-B) are set to $10^{-4}$. The PL model in [68] is adopted. Besides, we select the following two most relevant algorithms as benchmarks: (i) DA system with conventional AN (DAconv-AN) [3], where AN is generated towards the null-space of IRs whereas its disruptive effect should be kept for jamming potential Eves. Antennas are geographically deployed and antenna selection is enabled. (ii) CA MISO system without antenna selection (CA-no-AS) [38], where AN can be utilized as constructive element at IRs. Antennas are co-located and antenna selection is disabled. We also apply the proposed algorithms to CA systems, which are referred as CA-imperfectprob, CA-imperfect-det, CA-unknown-prob and CA-unknowndet respectively in simulation, and the multiple antennas of $\mathrm{CA}$ systems are co-located in the map center.

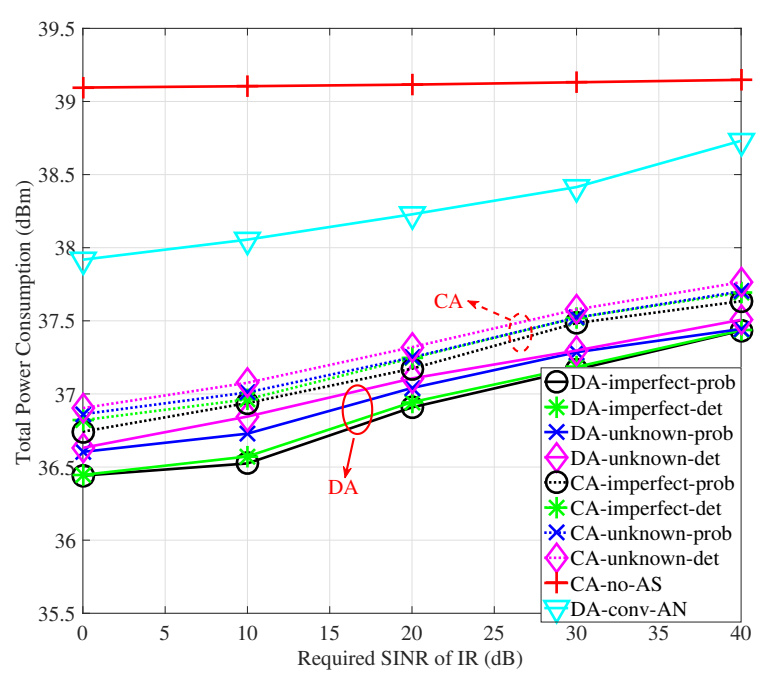

Fig. 5. Impact of the IR's SINR requirement $\bar{\Gamma}_{d}$ on the total power consumption, where $\bar{\Gamma}_{k}=-10 \mathrm{~dB}, \eta_{d}=\eta_{k}=0.95$, and $p_{A N}=25$ $\mathrm{dBm}$.

\section{A. Proposed Algorithms vs Benchmarks}

Fig. 5 shows the impact of the IR's SINR requirement $\bar{\Gamma}_{d}$ on the total power consumption with a random IR and Eve deployment. Firstly, it can be seen that the proposed algorithms outperform the two benchmarks, namely CA-no-AS and DAconv-AN. It is because the proposed algorithms benefit from DAs' activation/deactivation, the DAs far from the devices may be deactivated for saving power. Hence, the proposed algorithms achieve a device-centric and on-demand network structure with higher degree of freedom over the CA-no-AS algorithm in [38]. Besides, our proposed algorithms rotate AN to make it constructive to the IR even with imperfect CSI, while AN is treated as an undesired element at the IR by conventional DA-conv-AN in [3]. Secondly, benefiting from geographical distribution of the DAs, the distances between the DAs and devices are shortened and the DA systems can always find near antennas to serve the devices. With the alleviated PL, DA can outperform its counterpart CA in terms of power efficient transmission. Thirdly, increasing the SINR $\bar{\Gamma}_{d}$ leads to higher power consumption, as more antennas become activated 

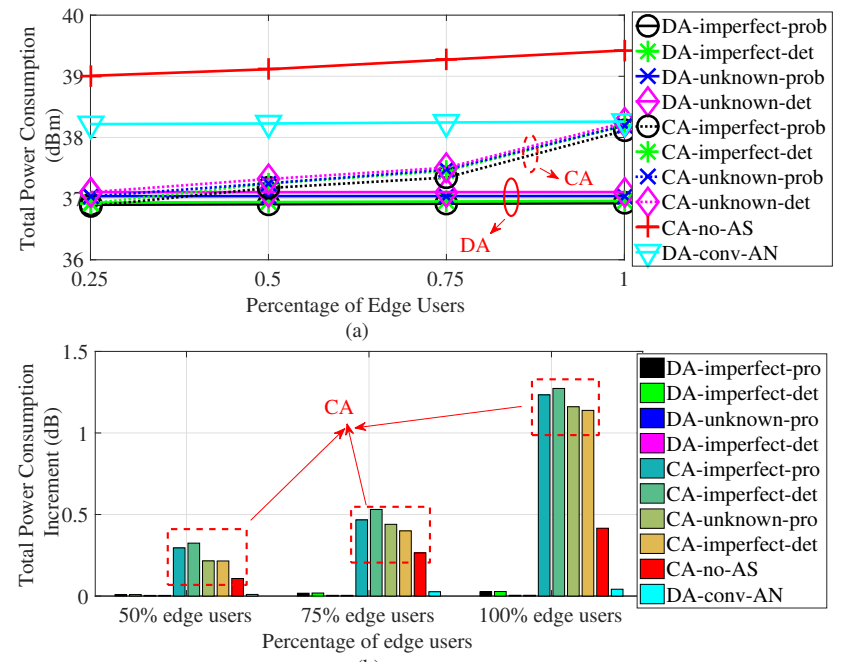

(b)

Fig. 6. (a) Impact of the percentage of edge users on power consumption (b) Power increment $(\mathrm{dB})$ of DA and CA systems with different percentages of edge users, where $\bar{\Gamma}_{d}=20 \mathrm{~dB}, \bar{\Gamma}_{k}=-10 \mathrm{~dB}, \eta_{d}=\eta_{k}=0.95$, and $p_{A N}=25 \mathrm{dBm}$.

and dissipate higher circuit and transmission power. Also, the power consumption of the CA-no-AS increases slowly as the increased transmission power is overwhelmed by its circuit power consumption. Fourthly, when the Eves' CSI is completely unknown, more power is dissipated compared to the scenario that the Eves' CSI is imperfectly obtained. It is because to address security, a minimum AN power level $p_{A N}$ is required, which is not efficient compared to the scenario that Eves' CSI is imperfectly obtained.

Fig. 6 (a) shows the total power consumption with different percentages of edge device. It demonstrates that with more devices at edge area, the power consumption of the DA system almost remains unchanged regardless of the devices' positions. It is because the geographically positioned DAs effectively extend the network coverage, and the proposed algorithms always activate near DAs for serving the devices. By contrast, the total power consumption of the CA systems keeps increasing when more devices move to edge area. This is because the CA systems have to activate more antennas and allocate higher transmission power to serve remote devices, which inevitably further improve the total power consumption of CA systems. On the other hand, Fig. 6 (b) shows the power consumption increment with different percentages of edge devices, benchmarked by the power consumption with $25 \%$ edge devices. It is obvious that the power consumption of the CA system increases significantly with more edge devices, and up to $1.3 \mathrm{~dB}$ increment of power consumption is achieved when all the devices are located in the edge area. Besides, it is worth noting that the power consumption increment of the CA-no-AS algorithm is lower than the other antenna selectionenabled CA systems, since the fully-activated antennas of the CA-no-AS have dominated the power consumption, which makes the increment less significant.

Fig. 7 shows the impact of the physical layer security requirement $\bar{\Gamma}_{k}$ on the total power consumption. Firstly, a

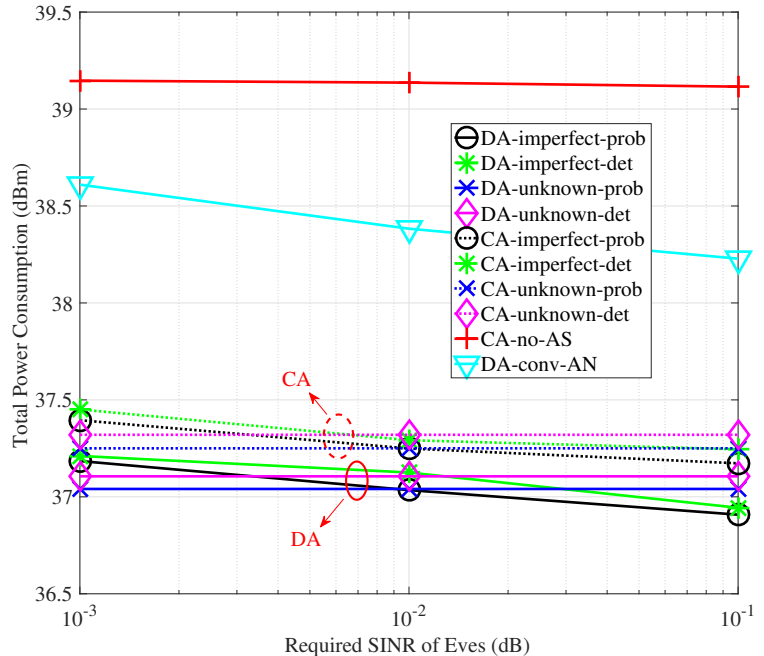

Fig. 7. Power consumption of DA and CA systems with different SINR requirement against the Eves, where $\bar{\Gamma}_{d}=20 \mathrm{~dB}, \eta_{d}=\eta_{k}=0.95$, and $p_{A N}=25 \mathrm{dBm}$.

stringent security constraint, such as $\bar{\Gamma}_{k}=10^{-3}$, leads to higher power consumption compared to a loose security constraint. It is because with a stringent constraint, higher power level of AN is needed to make the Eves' SINR lower than the requirement $\bar{\Gamma}_{k}$. Secondly, since the proposed algorithms efficiently utilize AN as a constructive element, the power consumption of the proposed algorithms maintains low and the security against the Eves can be simultaneously addressed. Thirdly, the DA systems always consume less power compared to the CA counterparts, benefiting from the device-centric structure. Fourthly, when the Eves' CSI is unknown at transmitter, the power consumption is independent with the value of $\bar{\Gamma}_{k}$. Hence, to address a stringent security requirement, one can properly increase the minimum power level of AN. That is, due to the channel disparity among the IR and Eves, the received signal of the Eves can be randomized in nature.

Fig. 8 shows the impact of CSI estimation error variance $\sigma_{d}^{2}$ and $\sigma_{k}^{2}$ on the probabilistic robust optimizations (DAimperfect-prob and DA-unknown-prob), and the impact of estimation error upper bound $\sigma_{d}^{U B}$ and $\sigma_{k}^{U B}$ on the deterministic robust optimizations (DA-imperfect-det and DAunknown-det). As can be seen, the power consumption of all the algorithms increases in a tough estimation scenario. In particular, for the probabilistic manner optimization, a higher CSI estimation error increases the norm of $\Theta_{d, 1}^{\frac{1}{2}}$ and $\Theta_{d, 2}^{\frac{1}{2}}$ and thus the left hand of the first inequality in Eq. (21) also increases, as suggested by Remark 1 . As a result, the amplitude of the precoder (also the transmission power) needs to be properly improved to make the optimization feasible, resulting in a increased total power. On the other hand, the deterministic manner robust optimization needs to keep the positive semidefinite characteristic for the matrices in Eq. (24), (25) and (27). This mathematically requires that all the leading principal minors in the matrices to be non-negative. Hence, with a higher CSI uncertainty, the amplitude of precoder (also the 


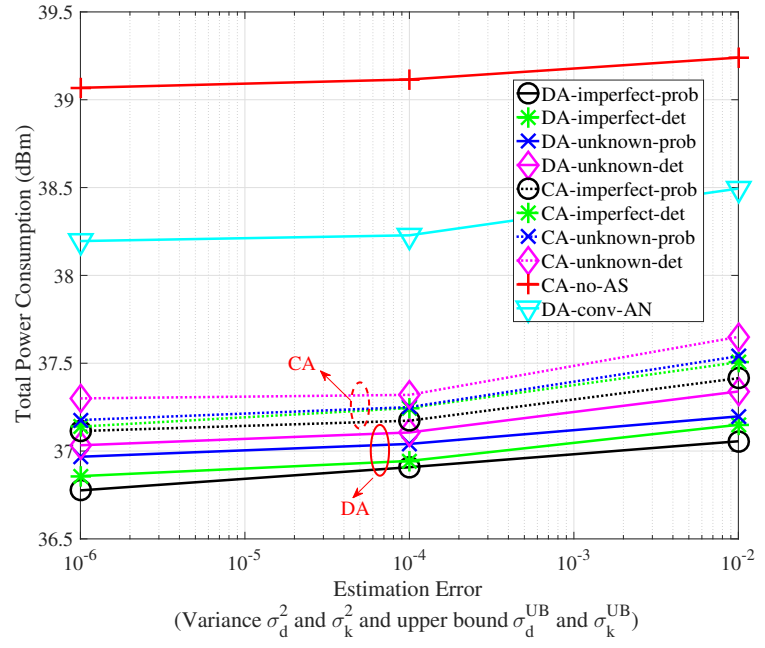

Fig. 8. Impact of the channel estimation error variance $\sigma_{d}^{2}$ and $\sigma_{k}^{2}$, and error upper bound $\sigma_{d}^{U B}$ and $\sigma_{k}^{U B}$ on the total power consumption, where $\bar{\Gamma}_{d}=20$ $\mathrm{dB}, \bar{\Gamma}_{k}=-10 \mathrm{~dB} \eta_{d}=\eta_{k}=0.95$, and $p_{A N}=25 \mathrm{dBm}$.

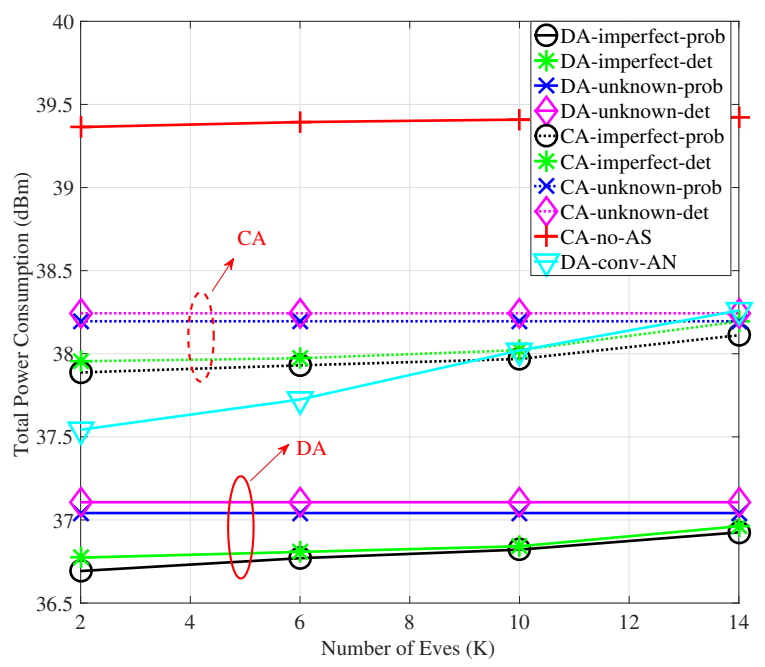

Fig. 9. Impact of number of the Eves on total power consumption, where $\bar{\Gamma}_{d}=20 \mathrm{~dB}, \bar{\Gamma}_{k}=-10 \mathrm{~dB}, \eta_{d}=\eta_{k}=0.95$, and $p_{A N}=25 \mathrm{dBm}$.

transmission power) needs to be property increased. As a result, the total power consumption of all the deterministic optimization is increased. A similar upwards trend can be also observed by the CA-no-AS and DA-conv-AN, which process the CSI uncertainties based on the deterministic manner as well.

Now, in the above Figs. 5-8, we have demonstrated that the proposed algorithms outperform the benchmarks. Importantly, it is clear from the above results that the proposed algorithms are most beneficial for edge users. Hence, in Fig. 9 and 10, we hereforth focus our attention on the scenario where all the IR and Eves are placed at the coverage edge to further highlight the advantages of DA deployment.

Fig. 9 shows how the total power consumption is affected by the number of Eves. Firstly, it can be seen that the DA de-
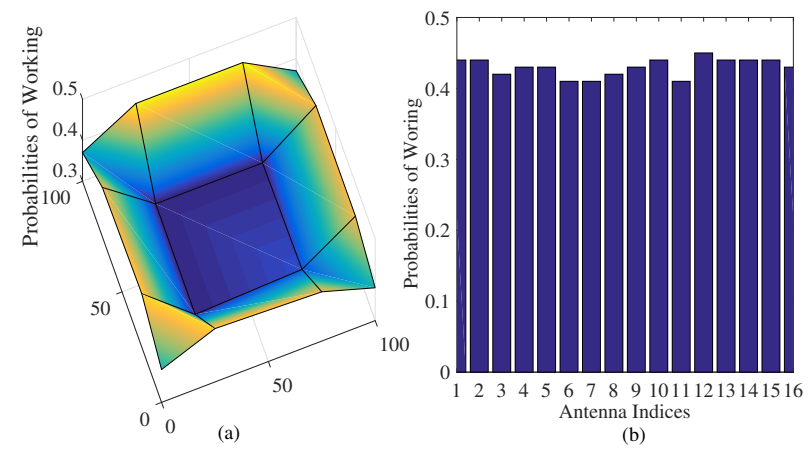

Fig. 10. (a) Impact of users' positions on antennas' modes in DA systems. (b) Impact of users' positions on antennas' modes in CA systems. $\bar{\Gamma}_{d}=20$ $\mathrm{dB}$ and $\bar{\Gamma}_{k}=-10 \mathrm{~dB} . \eta_{d}=\eta_{k}=0.95$, and $p_{A N}=25 \mathrm{dBm}$.

ployment always outperforms its CA counterpart regardless of the number of the Eves. For the antenna selection-enabled CA systems, they almost dissipate the same power compared to the DA-conv-AN benchmark. Although AN can be utilized by the antenna selection-enabled CA systems, the systems have to allocate higher transmission power for compensating severe PL, which is exactly avoided by the DA-conv-AN benchmark. As a result, the advantage of utilizing artificial noise vanishes in the antenna selection-enabled CA systems, especially in the scenario that all the IR and Eves are located at edge area. Secondly, higher power for generating $\mathrm{AN}$ is required to address the physical layer security when the number of the Eves increases, and thus higher total power consumption is led. Applying the concept of constructive AN yet pushing the IR's received symbols to the constructive region, the weight of precoder $\boldsymbol{w}$ can be interestingly reduced. As a result, the total power consumption of the proposed algorithms increase slowly with more Eves. By contrast, the two benchmarks dissipate more power with the increased number of the Eves, either hindered by high circuit power consumption at the multiple antennas (CA-no AS) or underutilized AN at the IR (DA-conv$A N)$. Especially for the DA-conv-AN scheme, since AN is treated as a harmful element at the IR, its power consumption increases significantly compared to other schemes. Thirdly, when the Eves' CSI is completely unknown by the system, the total power consumption remains unchanged with the increased number of Eves. However, one may preset a higher power level of $p_{A N}$ to address the physical layer security against the Eves, at the cost of high power consumption.

Fig. 10 shows how antennas' modes (activation or deactivation) are affected by the positions of the devices. For illustration, we only take the DA-imperfect-prob algorithm as an example due to the space constraints. It can be seen from Figs. 10 (a) that by the proposed algorithms, those DAs close to the devices have higher probabilities of working while the central DAs far from the devices have higher probabilities of being deactivated to save power. This is originated that in terms of power efficient design, letting those DAs far from the devices transmit signal is not power efficient. By contrast, as can be seen in Fig. 10 (b), the antennas in CA systems have the same probabilities of being activated or deactivated. 

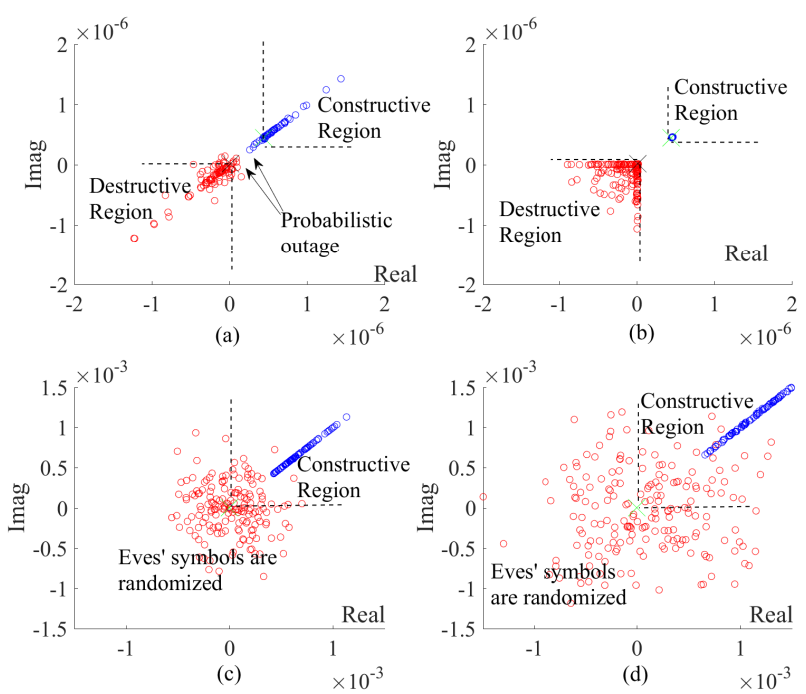

Fig. 11. Distribution of received signals on complex plane of the (a) DA-imperfect-prob, (b) DA-imperfect-det, (c) DA-unknow-prob, (d) DAunknown-det algorithms. X-coordinate and Y-coordinate denote the real and imaginary parts on complex plane, corresponding to that in Fig. 2. Red dots denote the received symbols of the Eves and blue dots denote the received symbols of the IR. Green cross denotes the IR's SINR requirement and black cross denotes the SINR constraint of Eves for addressing security. $\bar{\Gamma}_{d}=20$ dB. $\bar{\Gamma}_{k}=-10 \mathrm{~dB}, \forall k \in K . \eta_{d}=\eta_{k}=0.95$ and $p_{A N}=25 \mathrm{dBm}$.

It is because the centralized antennas have similar PL to the devices, and their working modes are insensitive to users' positions.

\section{B. CI Regions and Convergence Behavior}

For the DA-imperfect-prob algorithm in Fig. 11 (a), the received symbols of the IR locate in the constructive region while those of the Eves fall in destructive region in a probabilistic manner, satisfying the preset outage probabilities $\eta_{d}$ and $\eta_{k}, \forall k \in K$. This verifies that even with DA selection mechanism to save power, the proposed algorithm successfully keeps AN constructive for the IR whereas destructive for the Eves. It is because the algorithm statistically guarantees the IR's SINR higher than $\bar{\Gamma}_{d}$ and the Eves' SINR lower than the security threshold $\bar{\Gamma}_{k}$. Fig. 11 (b) shows the probabilities by the DA-imperfect-det algorithm. According to our analysis in section III-B, the IR' SINR and Eves' security constraints should be satisfied with all the CSI uncertainties. This is verified by the simulation that the received symbols of the IR locate in the constructive region while those of the Eves locate in the destructive region all the time, which is essentially different from the probabilistic optimization that allows proper outage occurs. However, as discussed in Fig. 5, this is achieved at the cost of high power consumption. Differently, Fig. 11 (c) and (d) show the probabilities when the Eves' CSI is unknown. Both probabilistic and deterministic robust optimization can locate the received symbols of the IR in the constructive region, which means the IR's SINR requirement is readily satisfied and AN is efficiently utilized. By setting the power level of AN higher than a threshold $p_{A N}$, the received symbols of the Eves are completely randomized, leading to a higher symbol error rate at the Eves.
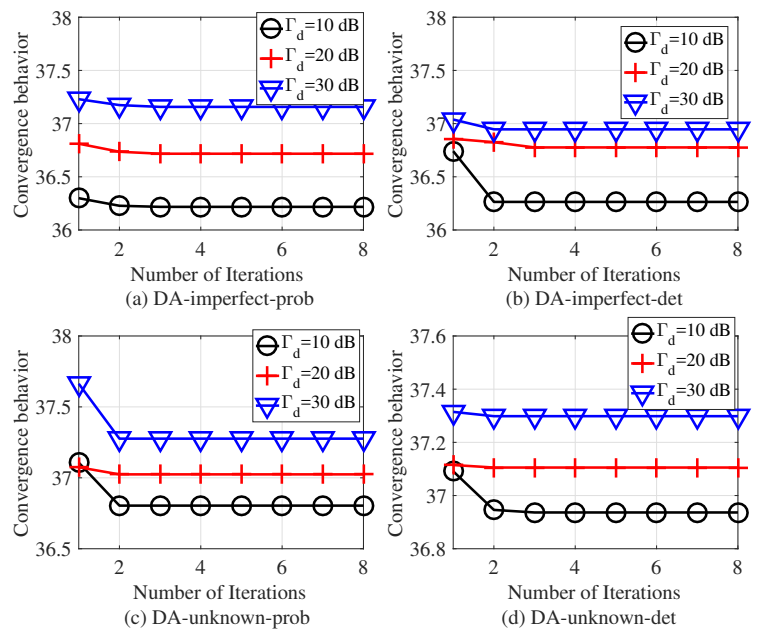

Fig. 12. Iteration behavior of the proposed algorithms, where $\bar{\Gamma}_{d}=20 \mathrm{~dB}$, $\bar{\Gamma}_{k}=-10 \mathrm{~dB}, \eta_{d}=\eta_{k}=0.95, \forall k \in K$, and $p_{A N}=25 \mathrm{dBm}$.

Fig. 12 shows the convergence of the proposed algorithms for updating antenna selection vector $\boldsymbol{t}$. It can be observed that the proposed algorithms are readily converged after 5 iterations, proving the low complexities of the algorithms.

\section{CONCLUSIONS}

We have investigated power minimization problem under physical layer security constraints for DA systems. Targeting at the two practical scenarios, DA selection vector and precoding have been jointly optimized in terms of probabilistic and deterministic robust optimizations. By the chance-constrained formulations as well as the deterministic formulations in the two robust optimizations, the proposed algorithms are able to satisfy the IR's QoS and simultaneously address physical layer security against the Eves. Our simulation results have showed that the proposed algorithms consume much lower power compared to the CA-no-AS and DA-conv-AN benchmarks, and low complexities have been confirmed by our computational analysis and simulation. Furthermore, a flexible and device-centric network structure is featured benefiting from antenna selection mechanism in DA systems, and the power consumption is maintained at low level regardless of the devices' positions compared to the CA counterpart.

\section{APPENDIX A}

The Essence of The Minimum Power Constraint on AN

In the conventional approaches, since the input signal is considered as Gaussian signal with infinite constellation size, the generated $\mathrm{AN}$ at potential Eves is assumed to be uncorrelated with the confidential signal. Hence, the AN at Eves is treated as noise and its effect is considered to be harmful to potential Eves. In contrast to the Gaussian input, practical transmission always has to rely on real-world modulation, which implicates a finite set of constellation points, and leads to finite-alphabet input. In this case, even $\mathrm{AN}$ is produced such that it lies in the null space of the receivers channel, the generated AN may 
indeed happen to be constructive to potential Eves. To clarify the essentiality of constraint (C20), let us write the $k$-th Eves channel in the form of $\boldsymbol{h}_{k}=\sqrt{\rho} \boldsymbol{h}_{d}+\sqrt{1-\rho} \boldsymbol{\epsilon}$. The parameter $\rho \in[0,1]$ measures the strength of the channel correlation between the IR and $k$-th Eves channels and $\boldsymbol{\epsilon} \in \mathcal{C N}\left(0, \boldsymbol{I}_{N}\right)$ is a random vector independent of $\boldsymbol{h}_{d}$ [69]. Evidently, $\rho=0$ means the IR and $k$-th Eves channels are un-correlated. Hence, the received signal at the $k$-th Eve can be equivalently written as

$$
y_{k}=\left(\sqrt{\rho} \boldsymbol{h}_{d}^{T}+\sqrt{1-\rho} \boldsymbol{\epsilon}^{T}\right) \boldsymbol{u} d e^{j \phi_{d}}+n_{k},
$$

where $\boldsymbol{u}=\boldsymbol{w}+\boldsymbol{z} e^{-j \phi_{d}}$ denotes the equivalent transmitting vector. The above equation can be further arranged into

$$
y_{k}=\sqrt{\rho} \boldsymbol{h}_{d}^{T} \boldsymbol{u} d e^{j \Phi_{d}}+\sqrt{1-\rho} \boldsymbol{\epsilon}^{T} \boldsymbol{u} d e^{j \phi_{d}}+n_{k},
$$

where the first term represents the symbol of interest while the second term is a random vector varying in each transmission. Hence, the random term can be utilized to randomize the distribution of the Eve received signal and thus degrade the detection performance of the Eves [69]. For this purpose, the power of $\boldsymbol{\epsilon}^{T} \boldsymbol{u}$ should be large enough to guarantee sufficient randomization to the phase of the Eves received signal. With unknown $\boldsymbol{\epsilon}$, we can place a constraint on the power of $\boldsymbol{u}$ instead. The average power of $\boldsymbol{\epsilon}^{T} \boldsymbol{u}$ can be approximated as $\mathbb{E}\left\{\left|\boldsymbol{\epsilon}^{\boldsymbol{T}} \boldsymbol{u}\right|^{2}\right\} \approx \operatorname{Tr}\left(\boldsymbol{u}^{H} \mathbb{E}\left\{\boldsymbol{\epsilon} \boldsymbol{\epsilon}^{H}\right\} \boldsymbol{u}\right)=\|\boldsymbol{u}\|^{2}$. Since we have $\boldsymbol{u}=\boldsymbol{w}+\boldsymbol{z} e^{-j \phi_{d}}$, imposing minimum power constraint on the AN $z$ has the same effect to that of the vector $\boldsymbol{u}$. Hence, the power constraint can be readily replaced by $\|\boldsymbol{z}\|^{2} \geq P_{A N}$, which randomizes the phase of the Eves received signal and hence deteriorates the Eves' symbol error rate performance.

\section{REFERENCES}

[1] Z. Wei and C. Masouros, "Robust secure precoding and antenna selection: a probabilistic optimization approach for interference exploitation," in Proc. ICASSP'19, Brighton, UK, May 2019.

[2] Z. Wei, C. Masouros, and F. Liu, "Interference exploitation based secure transmission for distributed antenna systems," in Proc. ICC'19, Shanghai, China, Jun. 2019.

[3] D. W. K. Ng and R. Schober, "Secure and green SWIPT in distributed antenna networks with limited backhaul capacity," IEEE Trans. Wireless Commun., vol. 14, no. 9, pp. 5082-5096, Dec. 2015.

[4] Z. Wei, S. Sun, X. Zhu, Y. Huang, and J. Wang, "Energy-efficient hybrid duplexing strategy for bidirectional distributed antenna systems," IEEE Trans. Veh. Technol., vol. 67, no. 6, pp. 5096-5110, Apr. 2018.

[5] X. Gao, O. Edfors, F. Tufvesson, and E. G. Larsson, "Massive MIMO in real propagation environments: Do all antennas contribute equally?" IEEE Trans. Commun., vol. 63, no. 11, pp. 39173928, Nov. 2015.

[6] Y. Huang and Y. Liu, "Energy efficient SWIPT in IoT distributed antenna systems, in IEEE Internet Things J., vol. 5, no. 4, pp. 2646-2656, Aug. 2018.

[7] Federal Communications Commission Order 11-50 (PDF), Accesse on: Sep. 25, 2014. [Online]. Available: https://apps.fcc.gov/edocspublic/attachmatch/FCC-11-50A1.pdf

[8] J. Wang and L. Dai, "Downlink rate analysis for virtual-cell based largescale distributed antenna systems," IEEE Trans. Wireless Commun., vol. 15, no. 3, pp. 19982011, Mar. 2016.

[9] C. Loyez, M. Bocquet, C. Lethien, and N. Rolland, "A distributed antenna system for indoor accurate WiFi localization," IEEE Antennas Wireless Propag. Lett., vol. 14, pp. 11841187, 2015.

[10] EARTH Project, "D2.3 Energy efficiency analysis of the reference systems, areas of improvements and target breakdown," Dec. 2010. [Online]. Available: http://www.ict-earth.eu/publications/publications.html

[11] J. Joung, Y. K. Chia, and S. Sun, "Energy-efficient, large-scale distributed antenna system (L-DAS) for multiple users," IEEE Trans. Signal Process., vol. 8, no. 5, pp. 964-965, Oct. 2014.
[12] Y. Shiu, S. Chang, H. Wu, S. Huang, and H. Cheng, "Physical layer security in wireless networks, A tutorial," IEEE Wireless Commun., vol. 12, no. 8, pp. 66-74, Apr. 2011.

[13] S. A. Sakoorian and A. L. Swindlehurst, "Optimal power allocation for GSVD-based beamforming in the MIMO wiretap channel," in Proc. ISIT'12., pp. 2321-2325, Boston, USA, 2012.

[14] H. Lei, J. Zhang, K. Park, P. Xu, and M. S. Alouini "Secrecy outage of max-min TAS scheme in MIMO-NOMA systems," in IEEE Trans. Veh. Technol., vol. 67, no. 8, pp. 6681-6690, Aug. 2018.

[15] H. Lei, H. Luo, K. H. Park, G. Pan, I. S, Ansari, and M. S. Alouini, "On secure transmission mixed RF-FSO system with TAS and imperfect CSI," arVix:1809.01503, Sep. 2018, [Online]: http://arvix.org/abs/1809.01503.

[16] S. Goel and R. Negi, "Guaranteeing secrecy using artificial noise," IEEE Trans. Wireless Commun., vol. 7, no. 6, 2180-2189, Jun. 2008.

[17] R. Negi and S. Goel, "Secret communication using artificial noise," in Proc. VTC Fall'15, Boston, USA, 2012.

[18] X. Zhou and M. R. Mckay, "Secure transmission with artificial noise over fading channels: achievable rate and optimal power allocation," IEEE Trans. Vech. Technol., vol. 59, no. 8, pp. 3831-3841, Oct. 2010.

[19] X. Zhang, X. Zhou, and M. R. Mckay, "On the design of artificial noise aided secure multi-antenna transmission in slow fading channels," IEEE Trans. Vech. Technol., vol. 62, no. 5, pp. 2170-2182, Jun. 2013.

[20] T. Zheng, H. Wang, J. Yuan, D. Towsley, and M. Lee, "Multi-antenna transmission with artificial noise against randomly distributed eavesdroppers," IEEE Trans. Wireless Commun., vol. 63, no. 11, 4347-4362, Nov. 2015.

[21] Q. Li and W. K. Ma, "Spatially selective artificial noise aided transmit optimization for MISO multi-eves secrecy rate maximization," IEEE Trans. Signal Process., vol. 61, no. 10, pp. 2704-2717, May 2013.

[22] W. Liao, T. Chang, W. K. Ma and C. Y. Chi, "QoS based transmit beamforming in the presence of eavesdropper: an optimized artificial noise-aided approach," IEEE Trans. Signal Process., vol. 59, no. 3, pp. 1202-1216, Mar. 2011.

[23] D. Wang, P. Ren, and J. Cheng, "Cooperative secure communication in two-hop buffer-aided networks," IEEE Trans. Commun., vol. 66, no. 3, pp. 972-985, Mar. 2018.

[24] Q. Li and L. Yang, "Artificial noise aided secure precoding for MIMO untrusted two-way relay systems with perfect and imperfect channel state information," IEEE Trans. Inf. Foren. Sec., vol. 13, no. 10, pp. 2628-2638, Oct. 2018.

[25] H. Lei, H. Zhang, I. S. Ansari, G. Pan, and K. A. Qaraqe, "Secrecy outage analysis for SIMO underlay cognitive radio networks over generalized-K fading channels," IEEE Signal Process. Lett., vol. 23, no. 8, pp. 1106-1110, Aug. 2016.

[26] K. Gao, Y. Gao, and G. Ascheid, "Security constraint power allocation in MU-massive-MIMO with distributed antennas," IEEE Trans. Wireless Commun., vol. 15, no. 12, pp. 8139-8153, Dec. 2016.

[27] H. Wang, C. Wang, D. W. K. Ng, M. H. Lee, and J. Xiao, "Artificial noise assisted secure transmission for distributed antenna systems," IEEE Trans. Signal Process., vol. 64, no. 15, pp. 4050-4064, Aug. 2016.

[28] A. Mukherjee and A. Swindlehurst, "Robust beamforming for security in MIMO wiretap channels with imperfect CSI," IEEE Trans. Signal Process., vol. 59, no. 1, pp. 351-361, Jan. 2011.

[29] C. Masouros and E. Alsusa, "A novel transmitter-based selective precoding technique for DS/CDMA systems," IEEE Sig. Process. Lett., vol. 14, no. 9, pp. 637-640, Sep. 2007.

[30] C. Masouros and E. Alsusa, "Dynamic linear precoding for the exploitation of known interference in MIMO broadcast systems," IEEE Trans. Wireless Commun., vol. 8, no. 3, pp. 1396-1404, Mar. 2009.

[31] C. Masouros, "Correlation rotation linear precoding for MIMO broadcast communications," IEEE Trans. Signal Process., vol. 59, no. 1, pp. 252262, Jan. 2011

[32] K. L. Law, C. Masouros, and M. Pesavento, "Transmit precoding for interference exploitation in the underlay cognitive radio Z-channel," IEEE Trans. Signal Process., vol. 65, no. 14, pp. 3617-3630, Jul. 2017.

[33] P. V. Amadori and C. Masouros, "Large scale antenna selection and precoding for interference exploitation," IEEE Trans. Commun., vol. 65, no. 10, pp. 4529-4542, Oct. 2017.

[34] A. Li and C. Masouros, "Interference exploitation precoding made practical: optimal closed-form solution for PSK modulations," Dec. 2017. [Online]. Available: https://arxiv.org/pdf/1712.07846.pdf

[35] M. Alodeh, S. Chatzinotas, and B. Ottersten, "Energy-efficient symbollevel precoding in multiuser MISO on relaxed detection region," IEEE Trans. Wireless Commun., vol. 15, no. 5, pp. 3755-3767, May 2016. 
[36] D. Spano, M. Alodeh, S. Chatzinotas, and B. Ottersten, "Symbol level precoding for the non-linear multiuser MISO downlink channel," IEEE Trans. Signal Process., vol. 66, no. 5, pp. 1331-1345, Mar. 2018.

[37] S. Timotheou, C. Masouros and I. Krikidis, "Exploiting constructive interference for simultaneous wireless information and power transfer in multiuser downlink systems," IEEE J. Sel. Areas Commun., vol. 34, no. 5, pp. 772-784, May. 2015

[38] M. Khandaker and K. Masouros, "Constructive interference based secure precoding: a new dimension in physical layer security," IEEE Trans. Inf. Foren. Sec., vol. 13, no. 9, pp. 2256-2268, Sep. 2018.

[39] Y. Sun, D. W. K. Ng, J. Zhu, and R. Schober, "Multi-objective optimization for robust power efficient and secure full-duplex wireless communication systems," IEEE Trans. Wireless Commun., vol. 15, no. 8, pp. 5511-5526, Aug. 2016.

[40] P. Gope and B. Sikdar, "Lightweight and privacy-preserving two-factor authentication scheme for IoT devices," IEEE Internet Things J., vol. 60, no. 1, pp. 580-589, Feb. 2019.

[41] P. Gope and B. Sikdar, "Privacy-aware authenticated key agreement scheme for secure smart grid communication," IEEE Trans. Smart Grid, vol. 14, no. 7, pp. 3953-3962, Jul. 2019.

[42] D. Kapetanovic, G. Zheng, and F. Rusek, "Physical layer security for massive MIMO: an overview on passive eavesdropping and active attacks," IEEE Commun. Mag., vol. 53, issue, 6, pp. 21-27, Jun. 2015.

[43] A. Mukherjee, S. A. A. Fakoorian, and A. L. Swindlehurst, "Principles of physical layer security in multiuser wireless networks: a survey," IEEE Commun. Surveys Tuts., vol. 16, no. 3, pp. 1550-1573, May. 2014.

[44] U. Maurer, "Secret key agreement by public discussion from common information" IEEE Trans. Inf. Theory, vol. 39, no. 3, pp. 733-742, May 1993.

[45] Y. Liu, H. Chen, and L. Wang, "Physical layer security for next generation wireless networks: theories, technologies, and challenges," IEEE Commun. Surveys Tut., vol. 19, no. 1, pp. 347-376. First quarter 2017.

[46] Y. Zou, J. Zhu, X. Wang, and L. Hanzo, "A survey on wireless security: technical challenges, recent advances, and future trends," Proceeding of IEEE, vol. 104, no. 9, pp. 1728-1765, Sep. 2016.

[47] K. J. Hole, E. Dyrnes, and P. Thorsheim, "Securing Wi-Fi networks," Computer, vol. 38, no. 7, pp. 2834, Jul. 2005.

[48] RFC 5246, "The transport layer security (TLS) protocol version 1.2," Aug. 2008. [Online]. Available: https://tools.ietf.org/html/rfc5246

[49] J. Zhang, T. Q. Doung, A. Marshall and R. Woods, "Key generation from wireless channels: a review," IEEE Access, vol. 4, pp. 614-626, Apr. 2016

[50] J. Zhang, A. Marshall, R. Woods, and T. Duong, "Efficient key generation by exploiting randomness from channel responses of individual OFDM subcarriers," IEEE Trans. on Commun., vol. 64, no. 6, pp. 25782588, Apr. 2016.

[51] Y. Liu, S. C. Draper, and A. M. Sayeed, "Exploiting channel diversity in secret key generation from multipath fading randomness," IEEE Trans. Inf. Forensics Security, vol. 7, no. 5, pp. 1484-1497, Oct. 2012.

[52] T. Aono, K. Higuchi, T. Ohira, B. Komiyama, and H. Sasaoka, "Wireless secret key generation exploiting reactance-domain scalar response of multipath fading channels," IEEE Trans. Antennas Propag., vol. 53, no. 11, pp. 3776-3784, Nov. 2005.

[53] N. Goergen, T. C. Clancy, and T. R. Newman, "Physical layer authentication watermarks through synthetic channel emulation," in Proc. IEEE Symp. New Front. Dyn. Spectr., Singapore, Apr. 2010, pp. 1-7.

[54] W. E. Cobb, E. D. Laspe, R. O. Baldwin, M. A. Temple, and Y. C. Kim, "Intrinsic physical-layer authentication of integrated circuits," IEEE Trans. Inf. Forensics Security, vol. 7, no. 1, pp. 14-24, Feb. 2012

[55] E. Martinian, G. W. Wornell, and B. Chen, "Authentication with distortion criteria," IEEE Trans. Inf. Theory, vol. 51, no. 7, pp. 2523-2542, Jul. 2005.

[56] A. Garnaev, M. B. Gursoy, and H. V. Poor, "A game theoretic analysis of secret and reliable communication with active and passive adversarial modes," IEEE Trans. Wireless Commun., vol. 15, no. 3, pp. 2155-2163, Mar. 2016.

[57] A. Mukherjee and A. L. Swindlehurst, "Jamming games in the MIMO wiretap channel with an active eavesdropper," IEEE Trans. Signal. Process., vol. 61, no. 1, pp. 82-91, Jan. 2013.

[58] Q. Zhu, W. Saad, Z. Han, H. V. Poor, and T. Basar, "Eavesdropper and jamming in next-generation wireless networks: a game-theoretic approach," in proc. of IEEE Military Communications Conference'11., Baltimore, USA, Nov. 2011.

[59] Y. Wang and R. Bagrodia, "ComSen: a detection system for identifying compromised nodes in wireless sensor networks," in proc. of IEEE
International Conference on Emerging Security Information, Systems and Technologies'12, Roman, Italy, Aug. 2012.

[60] R. Tandon, S. Amuru, T. C. Clancy and R. M. Buehrer, "Towards optimal secure distributed storage systems with exact repair," 2013, [Online]. Available: arXiv: $1310.0054 \mathrm{v} 1$.

[61] D. Nguyen, L. N. Tran, and M. L. Aho, "Precoding for full duplex multiuser MIMO systems: Spectral and energy efficiency maximization," IEEE Trans. Signal Process., vol. 61, no. 16, pp. 4038-4050, Aug. 2013.

[62] D. W. K. Ng, Y. Wu, and R. Schober, "Power efficient resource allocation for full-duplex radio distributed antenna networks," IEEE Trans. Wireless Commun., vol. 15, no. 4, pp. 2896-2911, Apr. 2016.

[63] Q. Wu, Y. Zeng, and R. Zhang, "Joint trajectory and communication design for multi-UAV enabled wireless networks," IEEE Trans. Wireless Commun., vol. 17, no. 3, pp. 2109-2121, Mar. 2018.

[64] S. Boyd and L. Vandenberghe, Convex Optimization. Cambridge, U.K. Cambridge Univ. Press, 2004

[65] E. Boshkovsha, D. W. K. Ng, N, and R. Schober, "Robust resource allocation for MIMO wireless powered communication networks based on a non-linear EH model," IEEE Trans. Commun., vol. 65, no. 5, pp. 1984-1999, Dec. 2017.

[66] K. Wang and C. Chi, "Outage constrained robust transmit optimization for multiuser MISO downlinks: tractable approximations by conic optimization,” IEEE Trans. Antenna Propagat., vol. 62, no. 21, pp. 56905715, Nov. 2014

[67] J. Zhang and J. G. Andrews,"Distributed antenna systems with randomness,"IEEE Trans. Wireless commun., vol. 7, no. 9, pp. 3636-3646, Sept. 2008.

[68] J. Pardo, J. Rodrguez, and L. Llcer, "Polarized Indoor MIMO channel measurements at $2.45 \mathrm{GHz}$," IEEE Trans. Antenna Propagat., vol. 56, no. 12, pp. 3818-3828, Dec. 2006

[69] Q. Xu, P. Ren, and A. L. Swindlehurst, "Rethinking secure precoding via interference exploitation: a smart eavesdropper perspective," 2019, [Online], Available: arXiv:1908.03174

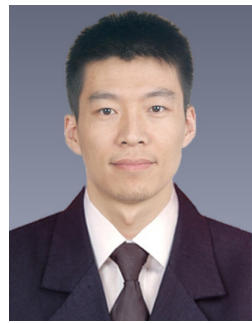

Zhongxiang Wei (S'15M'17) is research associate of Electrical and Electronics Engineering at University College London (UCL), U.K. He received the Ph.D. degree in Electrical and Electronics Engineering from the University of Liverpool (UOL), Liverpool, U.K., in 2017. From March 2016 to March 2017, he was with the Institution for Infocomm Research, Agency for Science, Technology, and Research (A*STAR), Singapore, as a Research Assistant. From March 2017 to October 2017, he was a visiting student with the Wireless Networks and Communications Group, Harbin Institute of Technology (HIT), Shenzhen, China.

His research interests include constructive interference design, green communications, full-duplex, millimeter-wave communications, and algorithm design. He was the recipient of the Graduate China National Scholarship Award in 2012, and the A*STAR Research Attachment Programme (ARAP) Studentship in 2016. He has acted as a TPC member or session chair for various international conferences, such as ICC 2019 and 2020. He was the recipient of exemplary reviewer of IEEE Transaction on Wireless Communications in 2016 


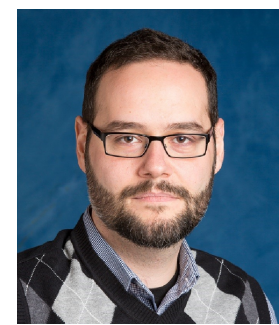

Christos Masouros (M' 06-SM' 14) received the Diploma degree in Electrical and Computer Engineering from the University of Patras, Greece, in 2004, and MSc by research and PhD in Electrical and Electronic Engineering from the University of Manchester, UK in 2006 and 2009 respectively. In 2008 he was a research intern at Philips Research Labs, UK. Between 2009-2010 he was a Research Associate in the University of Manchester and between 2010-2012 a Research Fellow in Queen's University Belfast. In 2012 he joined University College London as a Lecturer. He has held a Royal Academy of Engineering Research Fellowship between 2011-2016.

$\mathrm{He}$ is currently a Full Professor in the Information and Communications Engineering research group, Dept. Electrical and Electronic Engineering, University College London. His research interests lie in the field of wireless communications and signal processing with particular focus on Green Communications, Large Scale Antenna Systems, Cognitive Radio, interference mitigation techniques for MIMO and multicarrier communications. He was the recipient of the Best Paper Awards in the IEEE GlobeCom 2015 and IEEE WCNC 2019 conferences, and has been recognised as an Exemplary Editor for the IEEE Communications Letters, and as an Exemplary Reviewer for the IEEE Transactions on Communications. He is an Editor for IEEE Transactions on Communications, and IEEE Transactions on Wireless Communications. $\mathrm{He}$ has been an Associate Editor for IEEE Communications Letters, and a Guest Editor for IEEE Journal on Selected Topics in Signal Processing issues Exploiting Interference towards Energy Efficient and Secure Wireless Communications and Hybrid Analog / Digital Signal Processing for HardwareEfficient Large Scale Antenna Arrays. He is currently an elected member of the EURASIP SAT Committee on Signal Processing for Communications and Networking. 